\title{
Low level image partitioning guided by the gradient watershed hierarchy
}

\author{
I.E. Pratikakis*, H. Sahli, J. Cornelis \\ Vrije Universiteit Brussel, ETRO-IRIS, Pleinlaan 2, B-1050 Brussels, Belgium
}

Received 9 February 1998; received in revised form 21 November 1998

\begin{abstract}
In this paper, we study the construction of hierarchies for gradient watersheds. The underlying analysis is based on the fact that the gradient image provides information with respect to local discontinuity and similarity. Two algorithms which utilize this type of information are analyzed. The first one is an extended version of the dynamics of contours and the second is the algorithm which considers the minimum cost path saliency between two adjacent regional minima. For both algorithms, a stopping criterion which defines and extracts automatically each hierarchical level is proposed. An important hint to determine which one of the two algorithms has a superior behavior is the domination of the profile type of the surface (convex or concave) formed by the gradient image. Tests are discussed on artificially generated and real images. The proposed bottom-up procedure provides an essential aid to the precise classification of anatomical objects when used together with a user-interface environment. (C) 1999 Elsevier Science B.V. All rights reserved.
\end{abstract}

\section{Zusammenfassung}

Im folgenden Beitrag werden wir die Konstruktion von Hierarchien für Wendepunkte untersuchen. Die zugrundeliegende Analyse beruht auf daß Gradientenbilder Informationen über lokale Diskontinuitäten und Ähnlichkeiten beinhalten. Es werden zwei Algorithmen untersucht, die diese Art von Information nutzen. Der erste ist eine erweiterte Version der Dynamik von Konturen und der zweite Algorithmus betrachtet minimale Sprünge der Kostenfunktion zwischen zwei benachbarten regionalen Minima. Für beide Algorithmen wird ein Abbruchkriterium vorgestellt, das automatisch jede hierarchische Stufe definiert und extrahiert. Ein wichtiger Hinweis dafür, welcher der beiden Algorithmen ein besseres Verhalten liefert, ist die Dominanz des Profiltyps der Oberfläche (konvex oder konkav), gegeben durch das Gradientenbild. Tests mit künstlichen und realen Bildern werden diskutiert. Die vorgestellte bottom-up Methode liefert eine entscheidende Hilfe zur präzisen Klassifizierung anatomischer Objekte, wenn sie im Zusammenhang mit einer Benutzerschnittstelle eingesetzt wird. (C) 1999 Elsevier Science B.V. All rights reserved.

\section{Résumé}

Dans cet article, nous étudions la construction de hiérarchies pour les lignes de partage des eaux du gradient. L'analyse sous-jacente repose sur le fait que l'image gradient fournit de l'information relative aux discontinuités et aux similarités

\footnotetext{
* Corresponding author. Present address: IRISA, Unité INRIA/CNRS, Projet VISTA, Campus de Beaulieu, 35042 Rennes Cedex, France. Tel.: +33 2998475 16; fax: +3329984 71 71; e-mail: ioannis.pratikakis@irisa.fr
} 
locales. Deux algorithmes qui utilisent ce type d'information sont analysés. Le premier est une version étendue de la dynamique des contours et le deuxième est un algorithme qui considère le chemin de coût minimum entre deux minima régionaux adjacents. Pour les deux algorithmes, nous proposons un critère d'arrêt qui définit et extrait automatiquement chaque niveau hiérarchique. Une indication importante pour déterminer lequel des deux algorithmes a un comportement supérieur est la détermination du type de profile de la surface (convexe ou concave) formée par l'image gradient. Nous décrivons des tests sur des images générées artificiellement et sur des images réelles. La procédure ascendante que nous proposons fournit une aide essentielle à la classification précise d'objets anatomiques lorsqu'elle est integrée dans un environnement utilisant un interface utilisateur. (C) 1999 Elsevier Science B.V. All rights reserved.

Keywords: Gradient watersheds; Hierarchical segmentation; Medical images

\section{Introduction}

A meaningful image segmentation groups the pixels into disjoint regions that consist of uniform components. Facing an absence of contextual knowledge, the only alternative which can enrich our knowledge concerning the significance of our segmented groups is the creation of a hierarchy guided by the knowledge which emerges from the superficial and deep image structure [9]. Furthermore, a hierarchy can be constructed either on a pixel-to-pixel $[1,10]$ or on a region-to-region basis $[2,11]$. In the present work, we deal with information which can be retrieved from the superficial structure of the image and we strive towards the study of constructing hierarchical methods with respect to a region-based approach, wherein the initial pixel grouping is guided by the principles of the watershed analysis [2].

Several advantageous characteristics are ensured by applying the watershed transformation: (i) watersheds form closed curves providing a full partitioning of the image domain (thus, we get a pure region-based segmentation which does not require any closing or connection of the edges); (ii) watersheds on the image of gradient modulus can play the role of a multiple point detector [15], thus treating well all cases of multiple regions which meet at the same multiple point; (iii) there is a 1-1 relationship between the minima and the catchment basins. Therefore, we can represent a whole region by its minimum. Due to these advantages, image segmentation based on the use of the watershed transformation has proved to be an efficient method under the condition that its main drawback, namely over-segmentation, has been elimi- nated. All the efforts towards the solution of the over-segmentation problem follow two basic tendencies. In the first one, the question which arises is how to select an appropriate set of markers which will be used as a guide for an exclusive selection of the regions $[2,4]$. Most of the times the complexity of the regions makes the marking procedure a very difficult task, sometimes impossible to solve. The second trend deals with the saliency of each subpart of the watershed lines $[3,16]$. This subpart is the common boundary of adjacent partitions and its valuation in terms of a specified saliency assigns a hierarchical structure in the image. The work which is reported here deals with the second trend. As far as the watershed transformation is used for segmentation, it is common knowledge that it is best to only apply it on the gradient magnitude of an image because then the gradient magnitude information will guide the watershed lines to follow the crest lines and the real boundaries of the objects will emerge. Therefore, from now on, we will refer to gradient watersheds, thus explicitly implying that we have retrieved the watershed lines from the modulus of the gradient image.

In this paper, we address the problem of reducing over-segmentation which is produced by the classical morphological watershed transformation. Our objective is to create a hierarchy among the gradient watersheds which preserves the topology of the initial watershed lines and extracts homogeneous objects of a larger scale. For this purpose, we present a segmentation scheme which consists of two modules. The first module is dedicated to valuate each contour arc with a salient measure, while the second module identifies the different hierarchical 
levels by using a stopping criterion. With respect to the salient measure module, we have taken into account two alternatives. The first one is the dynamics of contours [16] while the other one is the saliency of the minimum cost path curve which traverses two adjacent regions [21]. The stopping criterion module employs statistical decision via hypothesis testing which enables an automatic retrieval of the hierarchical levels for different degrees of homogeneity. The proposed segmentation scheme extends and improves the dynamics of contours algorithm. Furthermore, in this paper, we examine the optimum cooperation of the two modules which depends on the selection of the algorithm for the contour valuation. For this purpose, we introduce a selection criterion which is based upon the domination of the profile type of the surface formed by the gradient image. This profile type is expressed by the bending of the minimum cost path curve between two adjacent regional minima, in the intensity dimension. For the sake of clarity, extensive tests have been applied on artificial and real images. Application has been demonstrated for a medical image analysis framework. The automatic retrieval of a small number of hierarchical levels can simplify scenarios used in an interactive environment for the precise definition of non-trivial anatomical objects.

The outline of the paper is as follows. Section 2 is dedicated to the notion of the grey-weighted distance which is the core of one of the algorithms that we propose for the contour valuation. We introduce a new tensor for its computation over discrete chamfer metrics and we briefly note a modification for the grey-weighted distance transform implementation in [28]. Section 3 provides a detailed description of the proposed hierarchical segmentation scheme. We discuss the contour valuation stage, the stopping criterion stage and the introduction of a selection criterion for attributing a preference for one of the two proposed contour valuation algorithms. In Section 4 we present and discuss segmentation results for artificial images and medical MR brain images along with a quantitative evaluation of segmentation quality with respect to noise. An application which uses the proposed segmentation scheme is described in Section 5. Finally, conclusions are drawn in Section 6.

\section{Grey-weighted distance}

Definition 2.1. Let $X \subset Z^{2}$ be a set and $x, y \in X$. The distance $d(x, y)$ between points $x$ and $y$ is defined as the length of the shortest path (if it exists and it is included in the set $X$ ) which connects those points. This length is also called geodesic distance. The set $X$ is called geodesic space.

The general formula for the geodesic distance is denoted as

$d(x, y)=\inf _{\Gamma(x, y)} \int_{s} \mathrm{~d} s$.

Regarding the line integral of Eq. (1) there is a consideration which plays a key role, namely the type of the arclength parameterization $s$. It is important to define the metric wherein our integration is computed. A general expression for the distance in any metric can be given by approximating the distance contribution ds from $\overrightarrow{\boldsymbol{x}}$ to $\overrightarrow{\boldsymbol{x}}+\boldsymbol{d} \overrightarrow{\boldsymbol{x}}$ by a straight line, using a metric tensor $g$ as explained in [25]:

$(\mathrm{d} s)^{2}=\sum_{i, j} g_{i j}(\overrightarrow{\boldsymbol{x}}) \mathrm{d} x_{i} \mathrm{~d} x_{j}$.

Therefore, Eq. (1) becomes

$d(x, y)=\inf _{\Gamma(x, y)} \int_{\gamma_{i j}} \sqrt{\sum_{i, j} g_{i j}(\overrightarrow{\boldsymbol{x}}) \frac{\partial x_{i}}{\partial t} \frac{\partial x_{j}}{\partial t}} \mathrm{~d} t$.

For the Euclidean metric we have

$g_{i j}(\overrightarrow{\boldsymbol{x}})=\delta_{i j}=\left[\begin{array}{ll}1 & 0 \\ 0 & 1\end{array}\right]$.

For the computation of distances over discrete chamfer metrics $(a-b)$ a new tensor $g_{i j}^{\prime}$ has to be defined as follows:

$g_{i j}^{\prime}=\left[\begin{array}{cc}\frac{2}{b^{2}} & \frac{1}{a b} \\ \frac{1}{a b} & \frac{1}{a^{2}}\end{array}\right]$,

where $a$ is the local distance between horizontal/vertical neighbors and $b$ is the local distance between diagonal neighbors [5]. A proof can be found in Appendix A. 
The grey-weighted DT has already been introduced in 1968 by Rutovitz [23]. According to this definition, the distance from a point to a set of reference points (the sources) is calculated as the lowest path sum of grey values (i.e. the lowest of the sums over all possible paths from that point to the sources). Let $V$ denote a set of prime vectors on the image sampling grid,

$V=\left\{\vec{v}_{1}, \ldots, \vec{v}_{m}\right\}$.

Let $Q$ denote a sequence of prime vectors $\vec{v}_{i} \in V$,

$Q=\left(\vec{v}_{i_{1}}, \ldots, \vec{v}_{i_{n}}\right), \quad 1 \leqslant i_{h} \leqslant m$.

The length $L$ of the path $Q$ is defined by the integration of local distances $d_{i}$ to the prime vectors $\vec{v}_{i}$ weighted by a cost function $w_{i}$ at point $i$. Using Eq. (4) for chamfer metrics and considering Eq. (3), we get

$L_{w}(Q)=\int_{\gamma_{i j}} w_{k} \sqrt{\sum_{i, j} g_{i j}^{\prime}(\overrightarrow{\boldsymbol{x}}) \frac{\partial x_{i}}{\partial t} \frac{\partial x_{j}}{\partial t}} \mathrm{~d} t$.

If the cost function $w_{i}$ is the grey value of the image itself, then the chamfer distance between two points $x$ and $y$ represented by the vectors $\vec{u}$ and $\vec{v}$ on the grid is defined as the minimum path length of all paths between $\vec{u}$ and $\vec{v}$ and is called grey-weighted distance.

$\operatorname{GWD}(\vec{u}, \vec{v})=\inf \left\{L_{w}(Q)\right\}$.

The implementation of the grey-weighted distance transform which we have used follows the 'write formalism' [28], where the minimization disappears and individual comparisons are applied as follows:

$\forall i:$ if $\left(p_{0}+c_{i}<p_{i}\right) \Rightarrow p_{i}=p_{0}+c_{i}$,

in which $p_{0}$ is the value of the central pixel, $p_{i}$ are the values of the neighboring pixels and $c_{i}$ are the corresponding chamfer distances. In our case, attention has to be paid to the distance valuation of the regional minima. This is due to the fact that at a regional minimum, either as a source or a destination region, the algorithm has to attribute the same distance to all the pixels which belong to the isovalued connected component. Therefore, we have modified the GWDT algorithm as it has been proposed in [28], to treat the source and destina- tion reference sets appropriately. An algorithmic description can be found in [19].

\section{Watershed analysis and hierarchical segmentation}

In this section, we present the notion of hierarchical segmentation which will be used to reduce over-segmentation produced by the classical watershed transformation. Our main goal is to create a hierarchy among the gradient watersheds which preserves the topology of the initial watershed lines and extracts homogeneous objects of a larger scale. Let us first define what we mean by a hierarchy.

Let $P^{0}=\left\{S_{1}^{0}, S_{2}^{0}, \ldots, S_{n}^{0}\right\}$ be the initial partitioning of the image $I$ due to the application of the watershed transformation. A hierarchical level $k\left(\mathrm{HL}_{k}\right)$ is defined as the partitioning $P^{k}=\left\{S_{1}^{k}, S_{2}^{k}, \ldots, S_{m_{k}}^{k}\right\}$ which preserves the inclusion relationship $P^{k} \supseteq$ $P^{k-1}$. This implies that each atom of the set $P^{k}$ is a disjoint union of atoms from the set $P^{k-1}$. A hierarchy is defined as a family which consists of all the hierarchical levels $\mathrm{HL}_{k}$, where $k \in[0, n]$ and $P^{n}=\{I\}$.

In the literature, different approaches exist for constructing a hierarchy of partitions defined on the basis of the watershed transformation and for retrieving information from the superficial image structure. In [12], partitioning the image in segmentation primitives is treated as a global optimization problem based on the minimum description length (MDL) of a criterion derived from attributes which describe similarity between regions. An updated hierarchy is provided, using the maximum current description length reduction as the indication of the merging at each iteration. Using this criterion in a global optimization problem, the produced hierarchy is lower bounded due to the global minimum, when it is reached. This property exhibits lack of flexibility with respect to a decrease of the image region cardinality. Hierarchical approaches emanating from a Mathematical Morphology (MM) framework consist of (i) the waterfall algorithm [2], (ii) the dynamics of minima [7] and (iii) the dynamics of contours [16]. This enumeration corresponds to the chronological order of their appearance in literature. Essentially, 
this hierarchy can be interpreted as a set of thresholded region adjacencies, wherein an ordering is determined by the value of the dynamics which are either controlled by the relative altitudes of the minima (dynamics of minima and dynamics of contours case) or by the relative heights of the watershed lines (waterfall case). A concise comparison of these approaches can be found in [3,16]. In [21], we propose an alternative to the above approaches. It examines the traversability of a path that traces the valley bottoms which connect two adjacent minima in the gradient image. Actually, this traversability can be expressed by the minimal cost to go from a minimum to its adjacent along all possible paths. The examination of this traversability points to the problem of the wavefront arrival time which can be solved by the grey-weighted distance transform [27] (see Section 2).

In this paper, we introduce a hierarchical segmentation scheme which consists of two stages. At the first stage we valuate the contour arcs with a salient measure and at the second stage we retrieve the different hierarchical levels by using a stopping criterion. In the sequel, we initiate the detailed discussion of the proposed scheme by describing the two algorithms which are going to be used for the contour valuation stage of our hierarchical segmentation scheme. These are the dynamics of contours [16] and the minimum cost path saliency [21].

\subsection{The contour valuation stage}

\subsubsection{Dynamics of contours}

The algorithm of dynamics of contours uses the principle of dynamics of minima as an initial information for the common contour valuation of adjacent regions. The dynamics of a minimum $m_{1}$ is easily defined with a flooding scenario. A unique source is placed at this minimum. As the altitude of the flood increases, neighboring catchment basins get flooded. Let $h$ be the altitude of the flood when for the first time a catchment basin with a deeper minimum $m_{2},\left(m_{2}<m_{1}\right)$ is reached. The dynamics of $m_{1}$ equals to $h$-altitude $\left(m_{1}\right)$. Since there exists a one-to-one correspondence between the minima and the catchment basins, the computation of dynamics of minima enables the valuation of the basin map. Each catchment basin is attributed the value of the dynamics of its minimum. Apart from the dynamics of minima, the additional information which is used for the computation of the dynamics of contours is based on the tracking of the flooding history. In such a way, a contour valuation can be found by the comparison of dynamics between the regions which have reached the contour of interest during a flooding. The contour valuation which is attributed to each common border of the regions is denoted as

$\operatorname{dyn}(a)=\min _{i} \max _{a_{i} \in B_{i}}\left\{f(a)-f\left(a_{i}\right)\right\}$

where $a$ denotes the lower point of the arc which belongs to the watershed and $B_{i}$ denotes an open connected component that belongs to the topological open set $\operatorname{Bas}(a)$ which is defined as

$$
\begin{gathered}
\operatorname{Bas}(a):=\{b \mid \exists \gamma, \gamma(0)=a, \gamma(1)=b, f(\gamma(s)) \\
<f(a) \forall s \in] 0,1]\} .
\end{gathered}
$$

For the sake of clarity, the computation of the dynamics of contours at the saddle or maximum point $P$ in Fig. 1 will be described. Suppose that we have already retrieved the dynamics of minima for

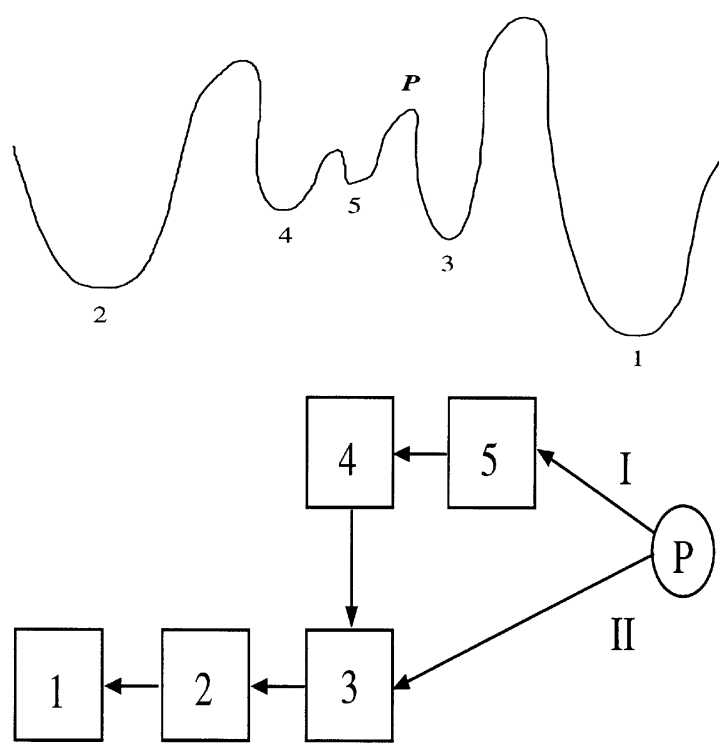

Fig. 1. Flooding list for point $P$. The basis for computing $D y$ namics of contours which corresponds to $P$. 
each catchment basin. During the flooding procedure we keep track for each catchment basin of the following information: (i) the dynamics of its corresponding minimum; (ii) the flooding chain of the catchment basins which have flooded it. Let $P$ be the point for which we want to identify the dynamics of contours. At the point $P$ we formally have a competition between the dynamics of catchment basins 3 and 5. Following a flooding procedure, it could be very natural that either catchment basin 3 or 5 has been flooded by another one and, therefore, the final competition at point $P$ turns out to be between stronger catchment basins with respect to their dynamics. An easy way to see which are the real competitive catchment basins is to look at the flooding chain. According to that chain, as it is depicted in Fig. 1, we have the following flooding facts. Catchment basin 5 has been flooded by 4, 4 has been flooded by 3, 3 has been flooded by 2 and in the end 1 is the catchment basin which floods all of them. These facts have been ordered according to the time that they occur. Using this flooding chain, we follow paths I and II starting from $P$ and identify the common node. In our example, it happens to be node 3 . The dynamics of contours at $P$ will be the maximum dynamics of minima between the catchment basins which precede node 3 in paths I and II. In our example, at path II there is no catchment basin which precedes 3 , while in path I there is catchment basin 4 . Therefore, the selected dynamics of minimum which will be attributed to $P$ is that of catchment basin 4 .

A shortcoming of this method is that retrieval of different levels of the hierarchy can be achieved only by applying a threshold. As an extension, we retrieve automatically the different hierarchical levels by applying a hypothesis test, as it is described in Section 3.2. This extended algorithm will be called constrained dynamics of contours.

\subsubsection{Minimum cost path saliency}

An important suggestion in favor of this approach is provided by the fact that traversing the gradient image provides useful information with respect to similarity between adjacent regions. For the sake of comparison, this traversal has been done in the same way for all the adjacent regions. Therefore, a reasonable choice for the path is the one which produces the minimum cost. The function $D_{F}(i, j)$ which measures this cost can be expressed as the minimization of the line integral of the local metric weighted by the local image intensity.

$D_{F}(i, j)=\inf \int_{\gamma_{i j}} F\left(\gamma_{i j}(s)\right) \mathrm{d} s$,

where $F=\|\nabla f\|^{2}$ is the squared gradient image, $f$ is the original image and $\gamma_{i j}(s)$ is any path between the regional minima $m_{i}$ and $m_{j}$ in the squared gradient image (Fig. 2), which correspond to the adjacent
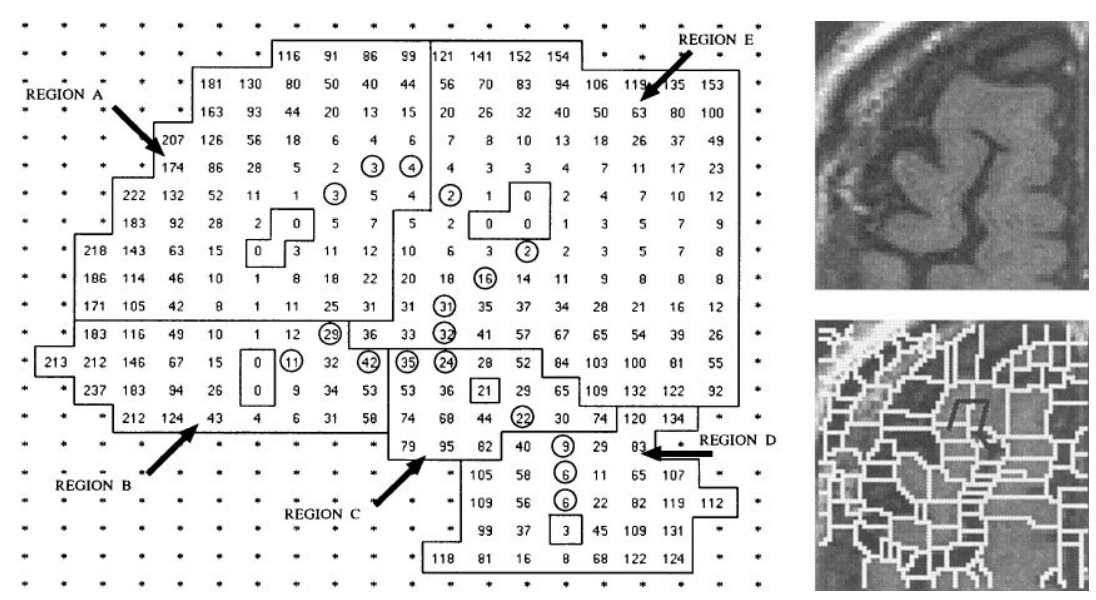

Fig. 2. The minimum cost path in the squared gradient image for adjacent regions. 
regions $O_{i}$ and $O_{j}$, respectively. By definition, the function $D_{F}(i, j)$ corresponds to the grey-weighted distance $G W D(i, j)$. The saliency measure which defines the closeness of two adjacent regions can be found by measuring the ratio of $D_{F}(i, j)$ to the generalized distance $\left(d_{F}^{\star}+1\right)$ [24]:

$S_{c_{i j}}=\frac{D_{F}(i, j)}{d_{F}^{\star}+1}$.

For the sake of clarity, we illustrate in Figs. 3 and 4 the computed GWDT maps which provide the value of $D_{F}(i, j)$ and the traced minimum cost path which provides the value of $d_{F}^{\star}$. Examples are given for the case that adjacent regions are homogeneous (Fig. 3) and inhomogeneous (Fig. 4). These maps are computed on the basis of the squared gradient image which is depicted as a height map in Fig. 2. This height map corresponds to the partitions which are traversed by the black line in the oversegmented image on the right of this map.
The nature of our line integral (Eq. (12)) is different from the one proposed in [15] which constituted the basis for the dynamics of contours. In [15] the use of the line integral expresses a measure of the work needed to move a point $a$ towards another point $b$, following a steepest descent path. For this purpose, the gradient vector field is used to combine the work calculation and the notion of topographic distance, as introduced in [13]. In our case, following the same topologically steepest path, we examine its traversability, weighting each point of this geodesic by the image value itself. The considered image value is the value of the squared gradient.

We have chosen to consider the squared gradient because the cost which is expressed by the greyweighted distance computation $\left(D_{F}\right)$ over the squared gradient image provides a better discrimination between the convex and concave path profiles of the gradient surface. This discrimination,

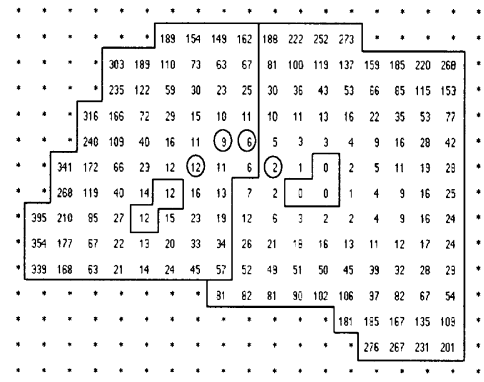

(a)

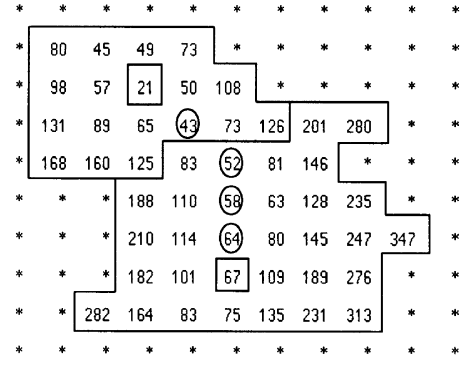

(b)

Fig. 3. GWDT maps for homogeneous adjacent regions: (a) adjacent regions (A,E); (b) adjacent regions (C,D).

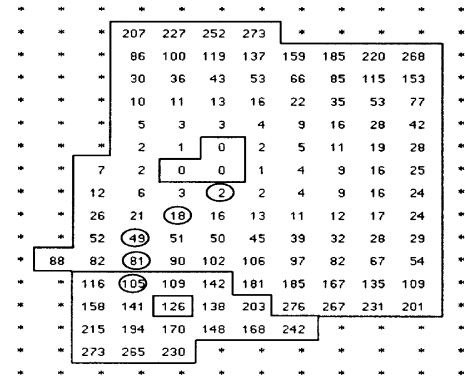

(a)

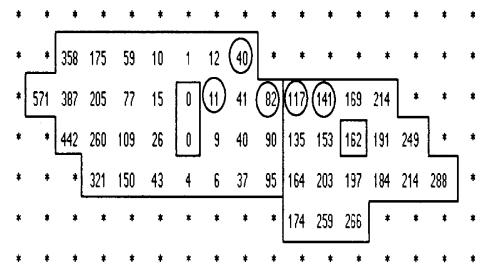

(b)

Fig. 4. GWDT maps for inhomogeneous adjacent regions: (a) adjacent regions (E,C); (b) adjacent regions (B,C). 
as it will be seen in Section 3.3, is important for estimating the homogeneity of the original image. For being consistent in our experiments, the squared gradient is also used for the dynamics of contours algorithm, although in this case it does not make any relative difference for the cost values.

The calculation of the saliency measure as defined in Eq. (13) along with the application of the stopping criterion stage, which will be described in Section 3.2, form the minimum cost path saliency algorithm.

\subsection{The stopping criterion stage - hypothesis testing in hierarchical segmentation}

When the calculation of the saliency measure is completed by using either Eq. (10) or Eq. (13), depending on the algorithm which is chosen, we apply a bubble sorting of all these values. As a result, we obtain the ranking of the adjacent region couples which are to be merged. From there on, the consecutive hierarchical levels can be constructed based on the ability of each merged couple to satisfy the null hypothesis during a hypothesis testing as we scan the ranked values. For our problem, the hypothesis set is defined as

$H_{0}^{k}$ : Two adjacent regions belong to the same label at level $k$.

$H_{1}^{k}$ : Two adjacent regions belong to different labels at level $k$.

Upon that definition it is critical to say that for every level transition from $k$ to $k+1$ we update the statistics used to formulate the hypothesis. This update is expressed by the error term $\sigma_{(\mathrm{err})}^{2}$. Considering that (i) our initial partitioning of the image (oversegmented image) consists of homogeneous regions and (ii) objects at a certain hierarchical level $k$ are expected to exhibit a certain homogeneity, we choose as the most suitable statistic the variance $\sigma^{2}$. Therefore, we formulate our hypothesis testing as follows:

$H_{0}^{k}: \quad \sigma_{\left(O_{i} \cup O_{j}\right)_{k}}^{2}=\tilde{\sigma}_{\left.(i)_{k}\right)}^{2}$,

$H_{1}^{k}: \quad \sigma_{\left(O_{i} \cup O_{j}\right)_{k}}^{2}>\tilde{\sigma}_{\left.(i)_{k}\right)}^{2}$,

where $\sigma_{O_{i} \cup O_{j}}^{2}$ denotes the variance of the merged region as a result of the updated regions $O_{i}$ and
$O_{j}$ and $\tilde{\sigma}_{(i j)_{k}}^{2}$ is expressed as

$\tilde{\sigma}_{(i j)_{k}}^{2}=\tilde{\sigma}_{\left(O_{i} \cup O_{j}\right)_{k}}^{2}+\sigma_{(\mathrm{err})_{k-1}}^{2}$,

where $\tilde{\sigma}_{\left(O_{i} \cup O_{j_{k}}\right.}^{2}$ denotes the expected variance of the merged region as a result of the updated regions $O_{i}$ and $O_{j}$. This is a result of weighting the variances of the updated constituent regions by the region area and it is equal to $\left(n_{i} \sigma_{i}^{2}+n_{j} \sigma_{j}^{2}\right) /\left(n_{i}+n_{j}\right)$. $\sigma_{(\mathrm{err})_{k-1}}^{2}$ denotes the error in variance at level $k-1$ due to which the alternative hypothesis was validated and consequently the hierarchical level $k-1$ was created. Initially, $\sigma_{(\mathrm{err})}^{2}$ is set to zero. Applying the chi-squared test to the calculated variance $s^{2}$ of the merged regions $O_{i}$ and $O_{j}$ we get

$s_{(i j)_{k}}^{2}<\frac{\tilde{\sigma}_{(i j)_{k}}^{2}}{n-1} \chi_{a}^{2}(n-1) \Rightarrow P(i, j)=$ true,

$s_{(i j)_{k}}^{2} \geqslant \frac{\tilde{\sigma}_{(i j)_{k}}^{2}}{n-1} \chi_{a}^{2}(n-1) \Rightarrow P(i, j)=$ false.

$P(i, j)$ is a decision function which is true if updated partitions $i$ and $j$ belong to the same object at level $k$, while otherwise it is false, and $n$ is the number of pixels in the merged region. Since in our case $n \gg 0$, we introduce the following approximation of the chi-square [17]:

$\chi_{a}^{2}(n-1) \approx(n-1)+u_{a} \sqrt{2(n-1)}$,

where $u_{a}$ is the right critical value for a one-tailed hypothesis test carried out on a standard normal distribution $(N(1,0))$. Substituting the approximation of Eq. (19) in Eqs. (17) and (18) leads to

$$
\begin{gathered}
\tilde{\sigma}_{(i)_{k}}^{2}\left(\frac{(n-1)+u_{a \sqrt{2(n-1)}}}{n-1}\right) \\
\approx \tilde{\sigma}_{(i j)_{k}}^{2}\left(1+\frac{u_{a \sqrt{2}}}{\sqrt{n}}\right) .
\end{gathered}
$$

Therefore, our test is expressed as

$$
\begin{aligned}
& s_{(i j)_{k}}^{2}<\tilde{\sigma}_{(i)_{k}}^{2}\left(1+\frac{u_{a \sqrt{2}}}{\sqrt{n}}\right) \Rightarrow P(i, j)=\text { true, } \\
& s_{(i j)_{k}}^{2} \geqslant \tilde{\sigma}_{(i j)_{k}}^{2}\left(1+\frac{u_{a \sqrt{2}}}{\sqrt{n}}\right) \Rightarrow P(i, j)=\text { false. }
\end{aligned}
$$

During all the experimental work, the confidence interval used was $a=0.05$, which corresponds to 
$u_{a}=1.96$. As a last note, it has to be mentioned that when we update the variances of the merged regions we do not have to compute them since statistics for a merged region can be computed by using statistics of the constituent partitions, which have already been computed at the very beginning.

\subsection{Convexity versus Concavity of the minimum cost path curve}

A gradient image provides information with respect to (i) local discontinuity (contrast) and (ii) local similarity (homogeneity for adjacent regions of the original image). The algorithm of constrained dynamics of contours uses contrast to construct a hierarchy for the image. On the other hand, the fact that local similarity can be retrieved from the gradient image is the basic premise in [21] for constructing a hierarchy. The actual information used is the averaging of the absolute gray values of the minimum cost path (valley bottoms) which connects two adjacent minima in the squared gradient image. This information guides the ranking of the saliency measures and is in agreement with the partial variance ordering of the ranked adjacent regions satisfying the null hypothesis in a hypothesis test. The degree of agreement between them is the basis for determining a meaningful hierarchy. A perfect agreement means that we retrieve hierarchical levels with emerging objects corresponding to those selected by a user. The relation between variance which reflects a certain degree of homogeneity and gradient information can be better perceived through the study of the minimum cost path curve profile shape in the intensity dimension. Convexities of this profile manifest an estimation of low variance, while concavities manifest an estimation of high variance. Fig. 5 and Table 1 illustrate the differences in variance which correspond to different shape path profiles for the same contrast.

Definition 3.1. Suppose that the function $f$ is differentiable at the point $a$ and that $L$ is the tangent line
Original Image

(a)

\begin{tabular}{|c|c|c|}
\hline 80 & 85 & $100+100,100$ \\
\hline 80 & 85 & $100,100,100$ \\
\hline 80 & 85 & $1001+001+100$ \\
\hline
\end{tabular}

A

(b)

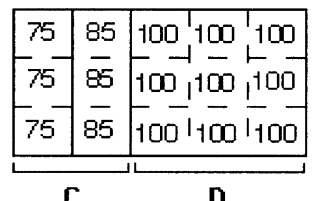

B
Gradient Image

\begin{tabular}{|c|c|c|c|c|}
\hline 0 & 5 & 15 & 0 & 0 \\
\hline 0 & $\frac{5}{5}$ & 15 & $\frac{0}{1}$ & 0 \\
\hline 0 & $\frac{5}{5}$ & 15 & 0 & 10 \\
\hline
\end{tabular}

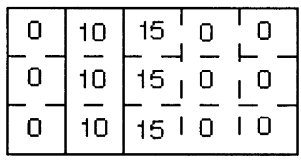

Path Profile
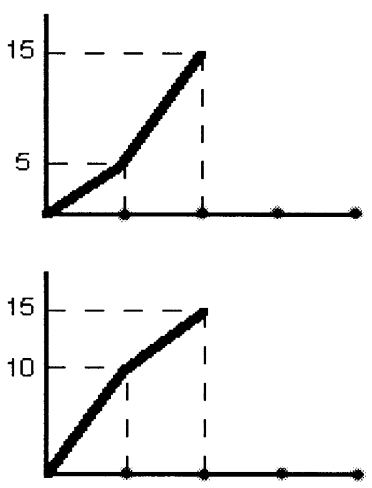

Fig. 5. Examples of (a) Convex and (b) Concave path profiles.

Table 1

Statistics wrt Convex/Concave path profiles

\begin{tabular}{lcrrrrr}
\hline Statistics/regions & A & B & C & D & A & B \\
\hline $\mathrm{n}$ & 6 & 9 & 6 & 9 & 15 & 15 \\
$\mu$ & 82.5 & 100 & 80 & 100 & 93 \\
$\sigma$ & 2.5 & 0 & 5 & 0 & 9.71 & 10.29 \\
$\sigma^{2}$ & 6.25 & 0 & 25 & 0 & $\mathbf{7 6}$ \\
\hline
\end{tabular}


to $y=f(x)$ at $(a, f(a))$. Then, the function $f$ (or its graph) is

(i) Convex at $a$ if on some open interval containing $a$, the graph of $f$ lies above $L$;

(ii) Concave at $a$ if on some open interval containing $a$, the graph of $f$ lies below $L$.

These two types of bending can be identified by checking the sign of the second derivative. Therefore, suppose that $f^{\prime}$ is differentiable on an open interval containing $a$. Then $f$ is: (i) Convex at $a$ if $f^{\prime \prime}(a)>0$; (ii) Concave at $a$ if $f^{\prime \prime}(a)<0$. The discrete correspondence of the second derivative at the point $a$ is denoted by the following three-point operator in one dimension:

$\nabla_{3}^{2} f=f_{a-1}-2 f_{a}+f_{a+1}$.

(a)
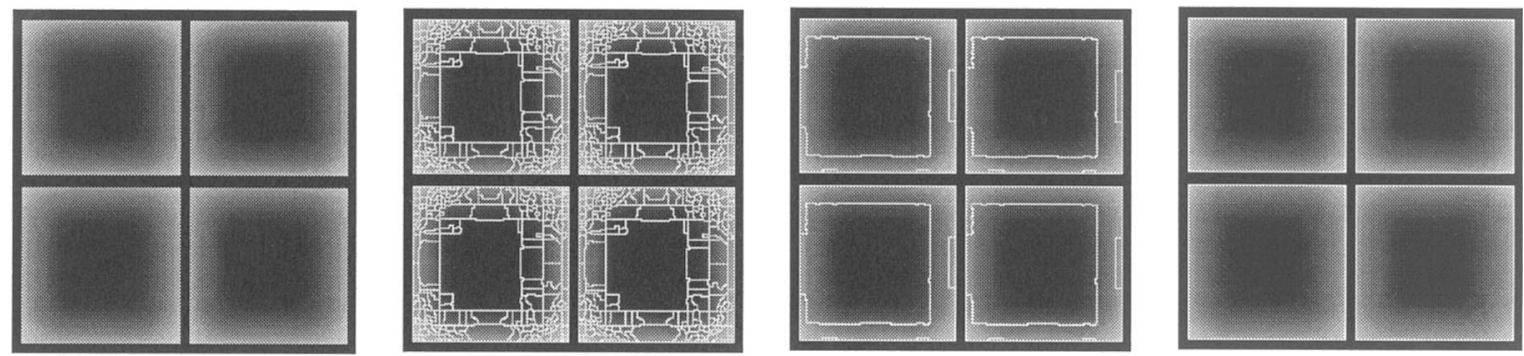

(b)
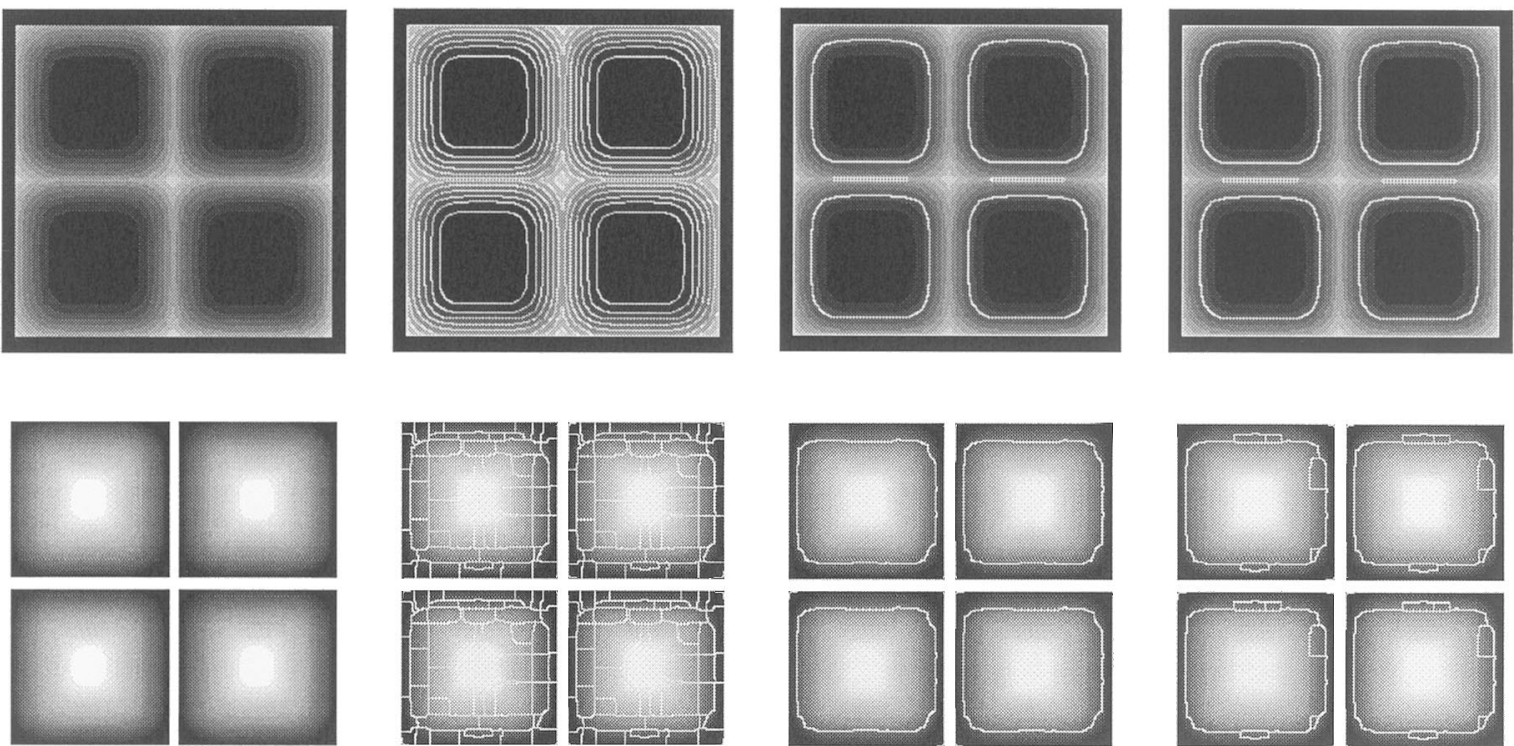

Fig. 6. Optimum hierarchical level in the case of (a) Concave domination, (b) Concave domination (ramp/step edges), (c) Convex domination. First column: Original image. Second column: Gradient watersheds. Third column: Optimum hierarchical level for DC algorithm. Fourth column: Optimum hierarchical level for MCPs algorithm. 
profiles (Fig. 6(c)). In case of concave path profile domination, algorithm MCPs constructs a hierarchy that can provide at its optimum level the best partitioning with respect to the degree of agreement. On the other hand, algorithm DC behaves better in case of convex path profile domination. A very interesting result is that of Fig. 6(b) which demonstrates the situation when the gradient image exhibits ramp/step edges. In this case, both algorithms provide exactly the same result. Figs. 7-10 demonstrate the complete hierarchical stack constructed by applying algorithms DC and MCPs on brain MR images. Slices were selected in such a way that both cases of convex and concave path profile
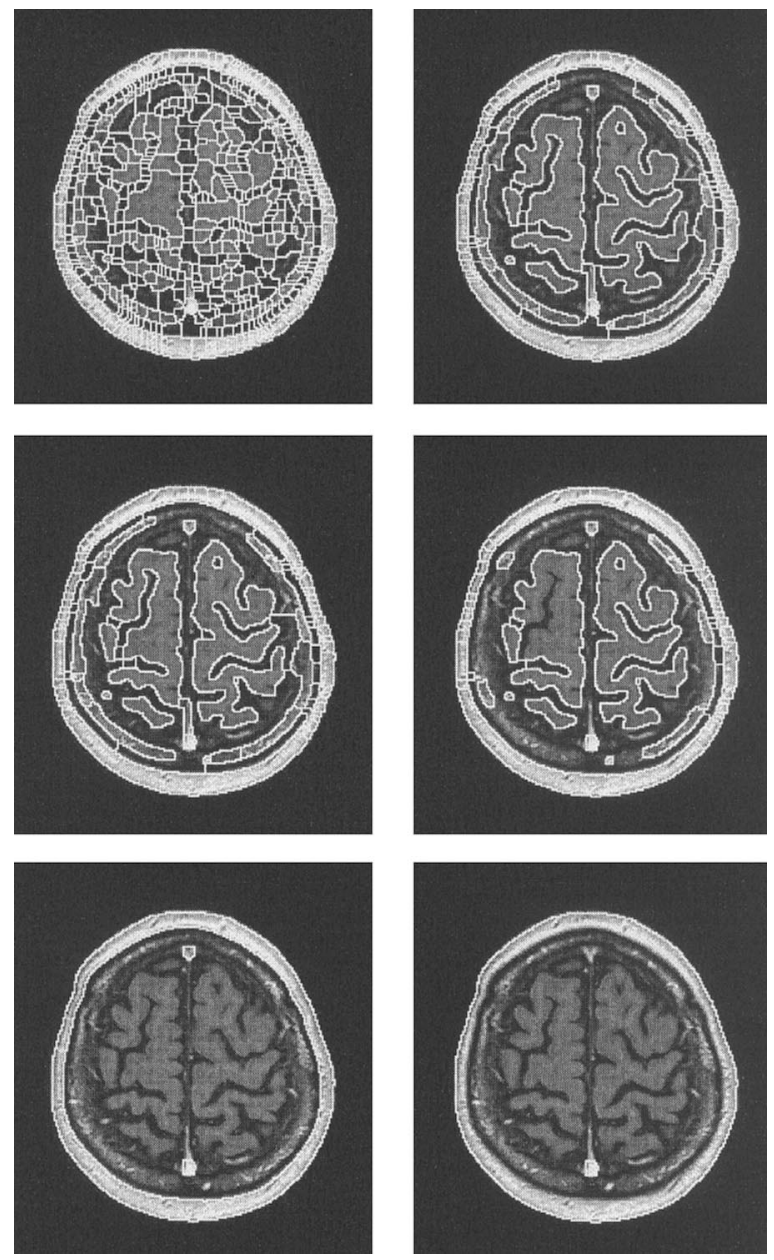

Fig. 7. Algorithm (DC) - complete hierarchical stack - Convex path profile domination. domination were tested. In these examples, we can again observe that for domination of concave path profiles the algorithm MCPs behaves better, while for convex path profile domination algorithm DC is superior.

\subsection{Quantitative evaluation of segmentation quality} with respect to noise

\subsubsection{Methodology}

The performance of (i) the constrained dynamics of contours (DC) and (ii) the minimum cost path saliency (MCPs) [21] hierarchical segmentation schemes, has to be examined with respect to noise. Such an evaluation can be realized by studying the behavior of both algorithms for a predefined input. For this purpose, a synthetic image has been generated containing two regions of different but constant grey levels. Those regions represent the background $(b)$ and foreground $(f)$ and their grey values are $g(b)=90$ and $g(f)=100$, respectively (Fig. 11). As a consequence the contrast $h$ between those regions is equal to $g(h)=10$. This ideal image is then corrupted by adding uncorrelated pseudorandom noise of both Gaussian and Uniform distributions. Our comparisons focus on the measure of the difference between the segmentation results of the initial image and its noisy counterpart for different degrees of noise corruption. In this context, the signal-to-noise ratio is defined as

$\mathrm{SNR}=\frac{h^{2}}{\sigma^{2}}$,

where $h$ is the contrast between the two regions and $\sigma^{2}$ is the variance of the additive noise.

A crude way to evaluate a segmentation result with respect to a predefined perfect segmentation is the counting of incorrectly labeled elements. This results in two probabilities (false positive and false negative) which do not consider any weighting for the misclassified labels. For that reason, we incorporate a method, initially introduced by Pratt [22], which was used for the evaluation of edge detectors. In our case, the application of the gradient watersheds results in a partitioning which is guided by the edges. Therefore, it is reasonable that we start from the same criterion as that of Pratt, but extend 

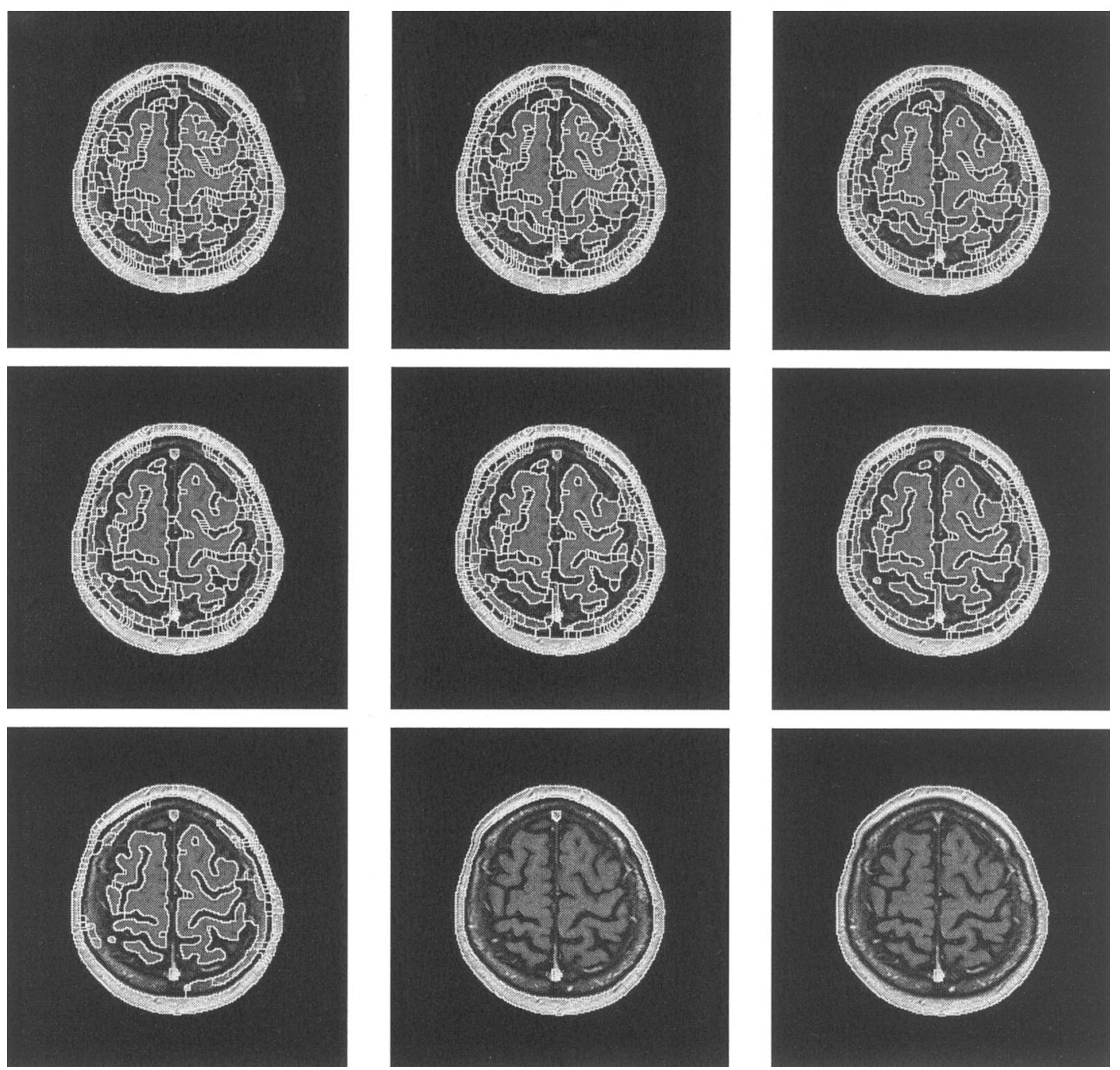

Fig. 8. Algorithm (MCPs) - complete hierarchical stack - Convex path profile domination.

it by taking into account regions (sets) rather than edges. We define the figure of merit (FOM) as

$\mathrm{FOM}=\frac{1}{\sum_{i=1}^{N} n_{i}} \sum_{i=1}^{N} \frac{n_{i}}{1+\gamma d^{2}(i)}$,

where $n_{i}$ is the number of pixels of region $i, d(i)$ is the minimum Euclidean distance between the set $i$ and its corresponding correct label and $\gamma$ is a scaling constant. This measure gives a value 1 to a cor- rect segmentation result. FOM is naturally considered as a spatial evaluation of the mislabelled sets. To incorporate a measure of grey value consistency inside the partitioned sets between the representative grey values of the segmented regions and their member image elements, we adapt the figure of certainty (FOC) [26] defined as

$$
\mathrm{FOC}=\frac{1}{\sum_{i=1}^{N} n_{i}} \sum_{i=1}^{N} \frac{n_{i}}{1+\left[\psi\left(f_{i}-\mu_{i}\right)\right]^{2}},
$$



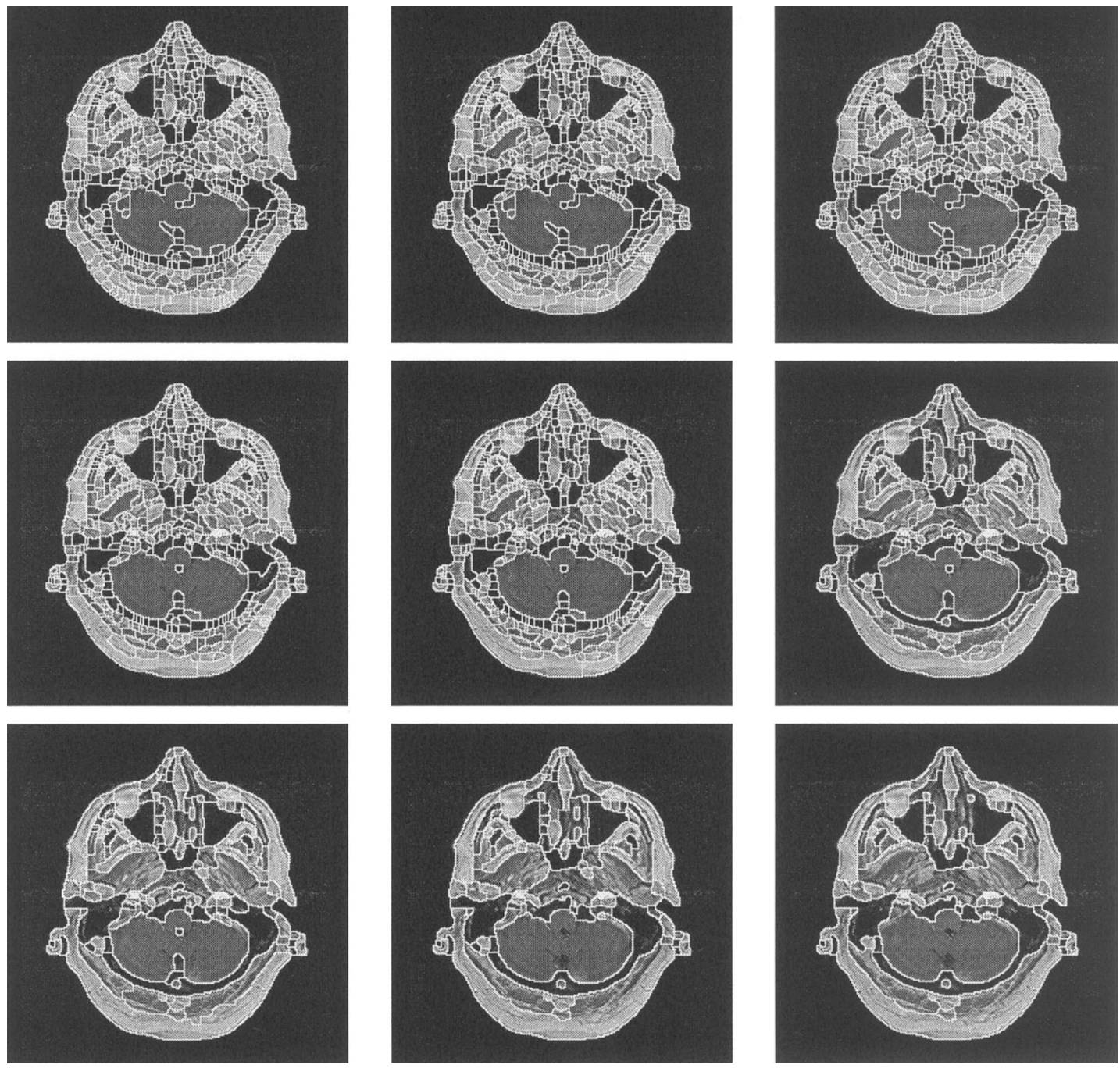

Fig. 9. Algorithm (DC) - complete hierarchical stack - Concave path profile domination.

where $n_{i}$ is the number of pixels of region $i, f_{i}$ is the grey value of region $i$ in the noise free test image, $\mu_{i}$ is the mean value of region $i$ and $\psi$ determines the contribution of the deviation from $f_{i}$. Here also, a correct segmentation gets a valuation of 1 . A last measure used for the quantitative evaluation of the proposed hierarchical schemes compares the actual numbers of regions found $n_{\mathrm{c}}$ against the true number $n_{\mathrm{tr}}$. It takes into account the fragmentation of the result and its values range from 0 to 1 . The variable $\tau$ is again a weighting factor.

$\mathrm{FRAG}=\frac{1}{1+\left|\tau\left(n_{\mathrm{c}}-n_{\mathrm{tr}}\right)\right|}$

\subsubsection{Discussion of results}

Corruption of the original image (Fig. 11) with both Gaussian and Uniform noise has been applied for variances which range from 6 to 20. The 

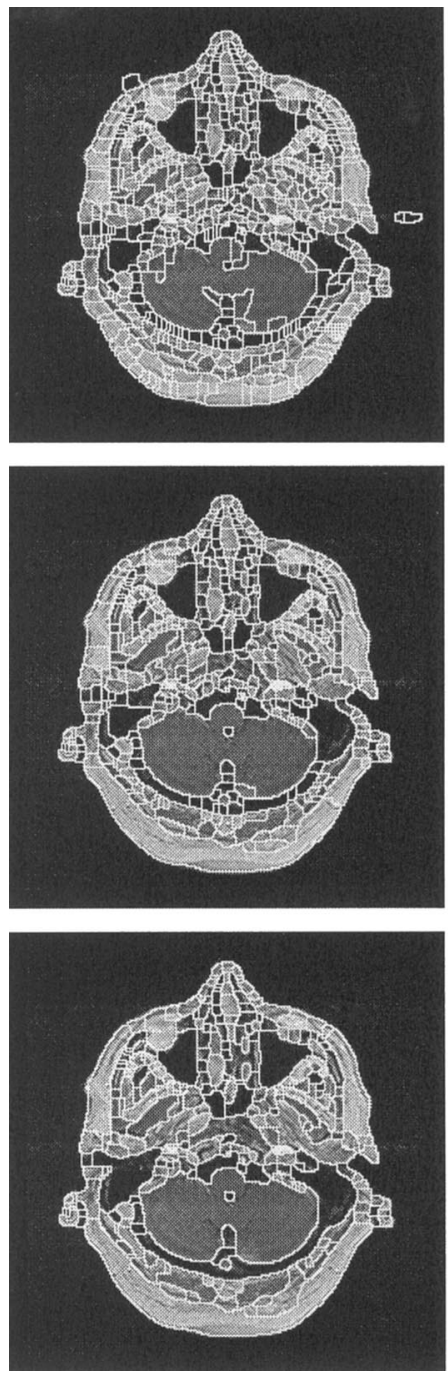
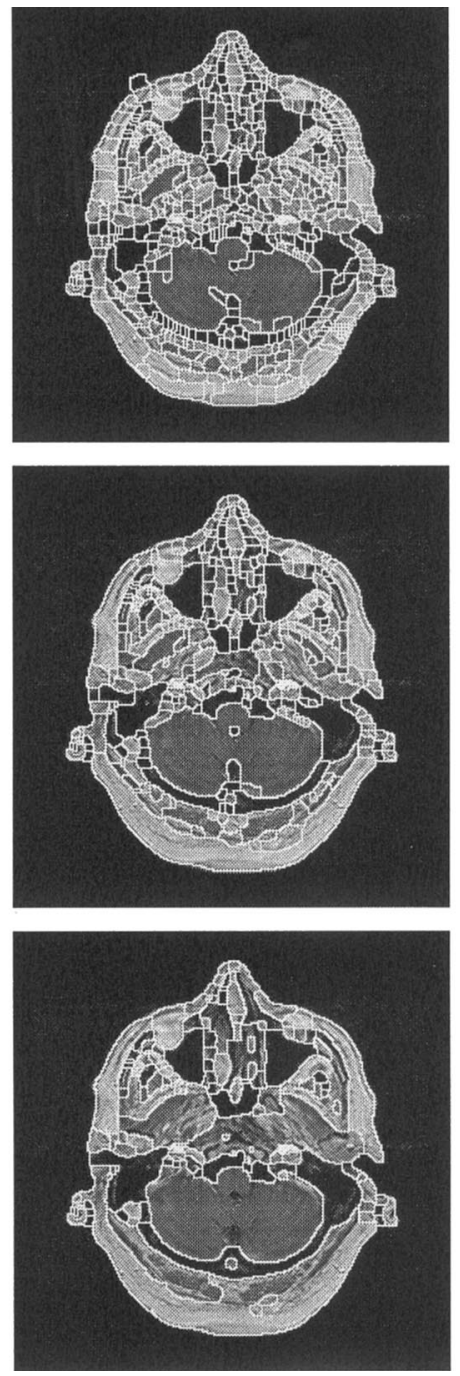
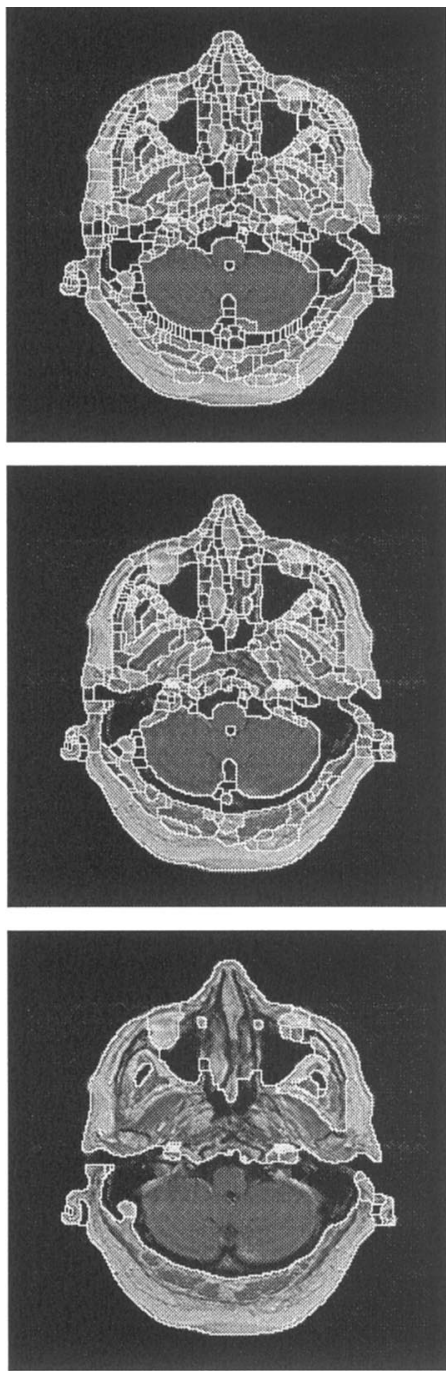

Fig. 10. Algorithm (MCPs) - complete hierarchical stack - Concave path profile domination.

algorithms DC and MCPs have been applied and the results are demonstrated in Fig. 12 for Gaussian noise and in Fig. 13 for Uniform noise. Figs. 14 and 15 contain graphs that aid the comparison of both algorithms with respect to the criteria FOM, FOC and FRAG. The scaling parameters of the measures expressed in Eqs. (25)-(27) have been fixed at the following values: $\gamma=0.8, \psi=0.16$ and $\tau=0.01$. The conclusion which can be extracted after all the experiments is that in all cases the MCPs algorithm exhibits the best performance for all the criteria. Although for the Uniform noise case its perfor- mance is similar to that of DC, in the Gaussian noise case it is clearly the best performant algorithm. These results were naturally expected due to the plethora of concave path profiles which are created in the presence of noise.

\section{Application: precise interactive delineation of 3D structures in medical images}

Automatic segmentation methods are known to be erroneous due to the complexity and variability 


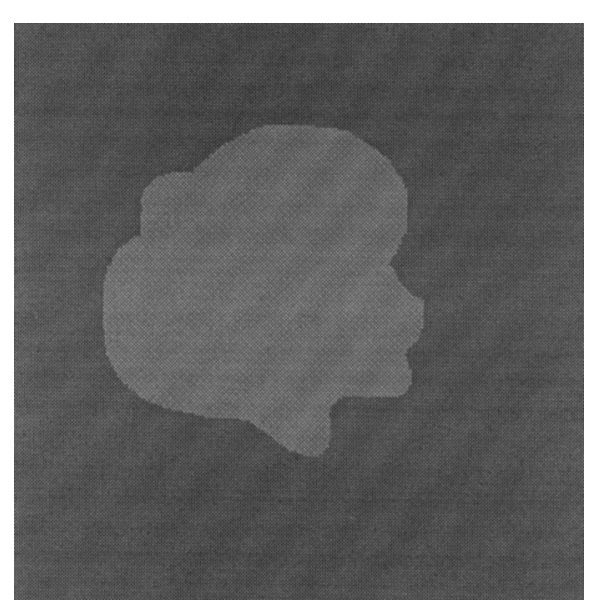

Fig. 11. Test image.

of medical images and cannot be applied without supervision by the user. On the other hand, manual segmentation is a tedious and time consuming process, lacking precision and reproducibility. Moreover, it is impractical when applied to extensive temporal and spatial sequences of images. Therefore, to perform an image segmentation task in a generic and efficient way, a compromise has to be found between automatic and manual modes. Under this principle, a hybrid scheme has to be constructed which minimizes the user input and allows the presence of intelligent tools to provide fast and accurate manual correction. The user input is minimized by constructing an image description rich in meaningful regions with low cardinality while an intelligent interactive tool ensures accuracy and reproducibility without requiring any special training by the user. A meaningful image description is obtained by following the reported hierarchy determination on an initial partitioning extracted by the gradient watersheds. Specifically, a combination is used between algorithms DC and MCPs. Whether the one or the other will be chosen is based on the type of the path profile (Concave/Convex) expressed by the bending of the minimum cost path curve between adjacent regional minima, in the intensity dimension. In the case that Convex path profiles dominate in number, DC is chosen and the contour arcs are valuated by the cost value $\operatorname{dyn}(a)$ which has been defined by Eq.
(10). On the other hand, if Concave path profiles dominate, MCPs is the algorithm which is chosen to create the hierarchy and the contour arcs are valuated by the cost value $S_{c_{i j}}$ which has been defined by Eq. (13). A concise description of the proposed hierarchical segmentation scheme is depicted in the schematic diagram of Fig. 16.

Similar efforts which utilize the watershed transformation have been reported by other authors. In [18] a system for interactive object definition has been implemented based on an automatically computed image description of sensible image regions obtained after successive blurring. This image description is a quasi-hierarchy of ridges and subridges in the intensity surface corresponding to the image. The same approach cannot be applied in our case because during successive blurring it can occur that watersheds which belong to the same ridge do not merge together before the parent ridge merges to a neighbor. In [12], partitioning the image in segmentation primitives is treated as a global optimization problem based on the minimum description length of a criterion derived from attributes which describe similarity between regions. An updated hierarchy is provided, using the maximum current description length reduction as the indication of the merging at each iteration. At a second stage the output image partitioning provides the input for an intelligent paintbrush tool. This approach lacks flexibility because it will always stop at the global minimum. Therefore, there is no way to merge further and compare different levels.

Instead, our proposed hierarchical segmentation scheme can help the application due to the automatic retrieval of a hierarchy which consists of a small number of levels, thus browsing through does not become a tedious task, and each of these levels may provide us meaningful delineations with respect to a certain degree of homogeneity. Following our approach, once the complete stack of all possible hierarchical levels has been calculated, the user can interact and intervene for the final precise delineation of the 3D structures [20]. Interactivity is applied in a 2D slice-by-slice fashion, reminiscent of the way that physicians work during a daily clinical routine. The scenario which is proposed is initiated by fast browsing through the different 

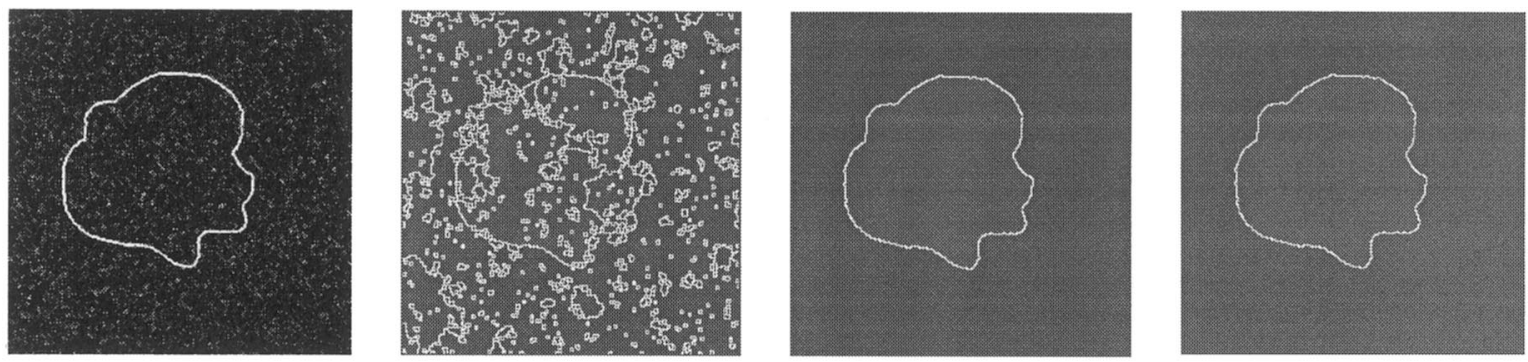

(b)
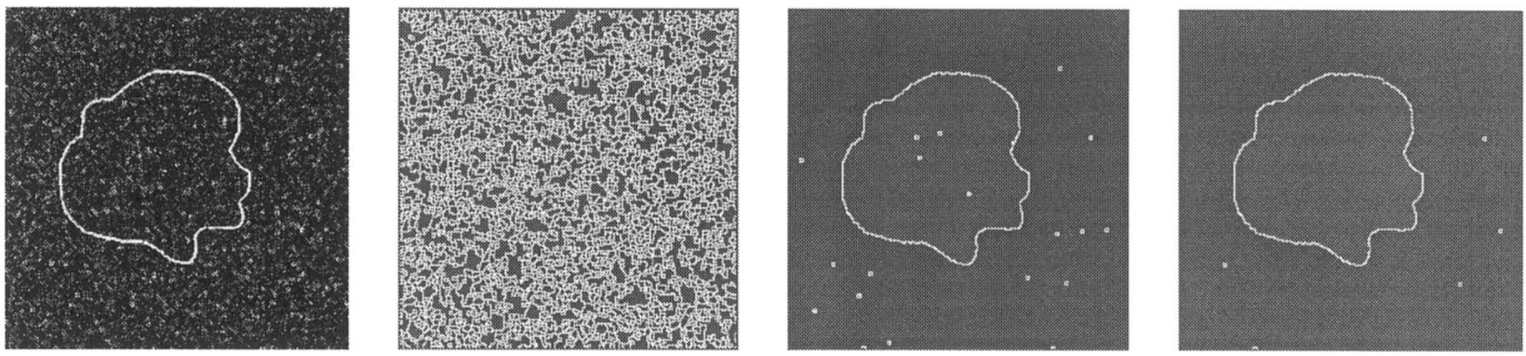

(c)
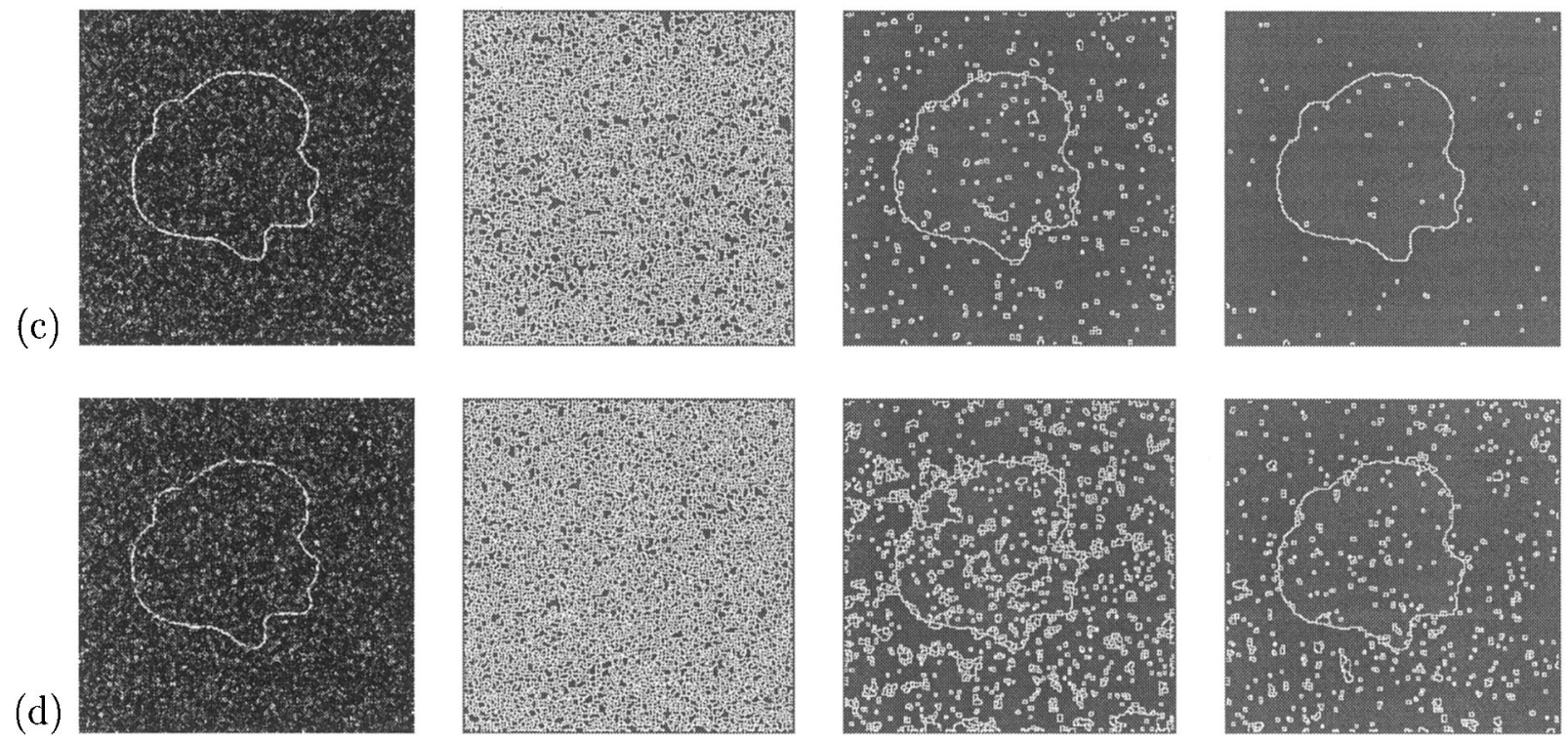

Fig. 12. Gaussian noise: (a) SNR $=12.2 \mathrm{db}\left\{\sigma^{2}=6\right\}$, (b) $\mathrm{SNR}=10.0 \mathrm{db}\left\{\sigma^{2}=10\right\}$, (c) $\mathrm{SNR}=8.2 \mathrm{db}\left\{\sigma^{2}=15\right\},(\mathrm{d}) \mathrm{SNR}=6.9 \mathrm{db}$ $\left\{\sigma^{2}=20\right\}$. First column: squared gradient image. Second column: oversegmented image. Third column: optimum level by applying algorithm (DC). Fourth column: optimum level by applying algorithm (MCPs).

hierarchical levels (see Fig. 17). This enables the user to select the optimal level in terms of minimal interaction.

The notion of interactivity entails two basic actions. The first action is the inclusion/exclusion of a segment and can be activated by a simple mouse click. In the case that segments have to be included which are not present, on-the-fly selection of a finer level out of the hierarchical set is feasible. The second action is the conversion of the aggregated 
(a)
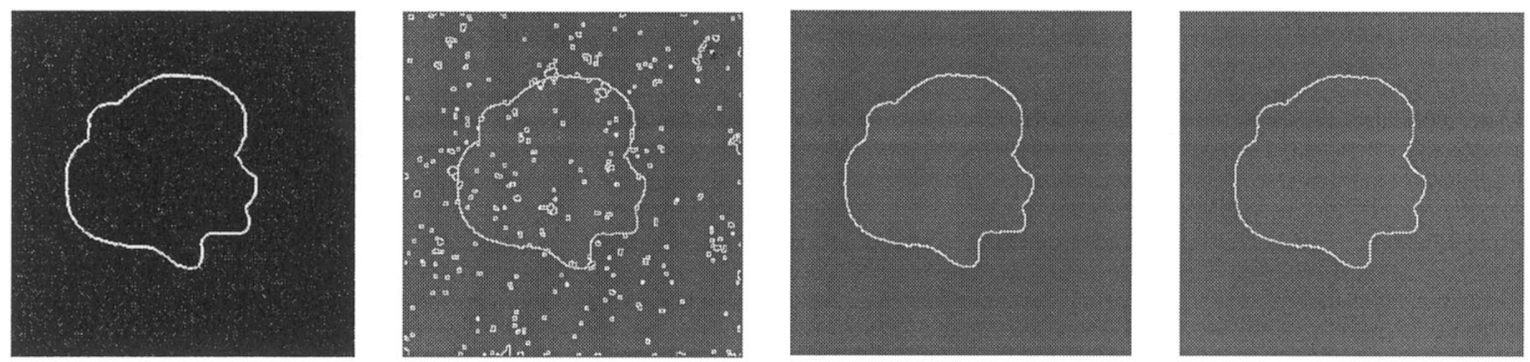

(b)
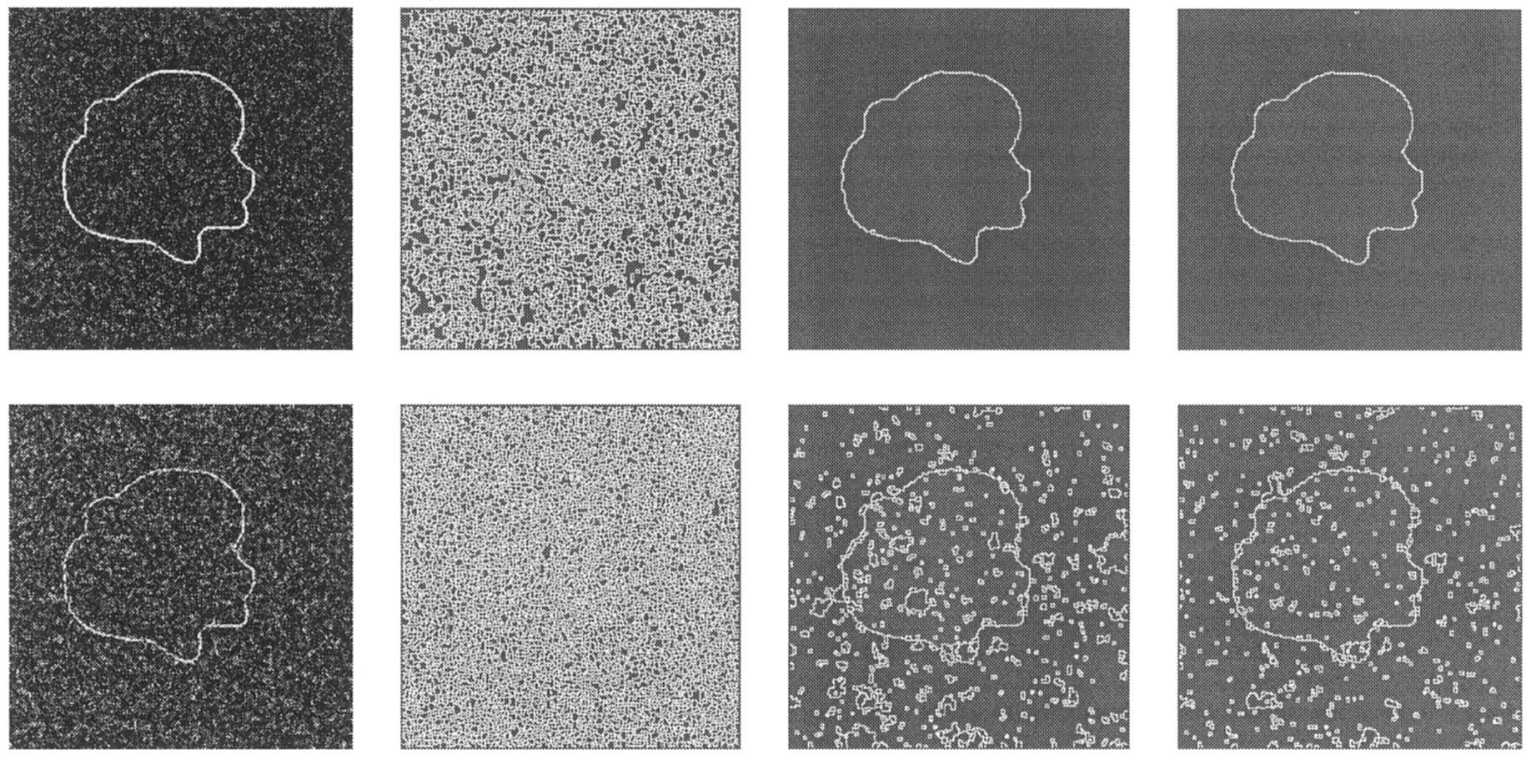

(c)
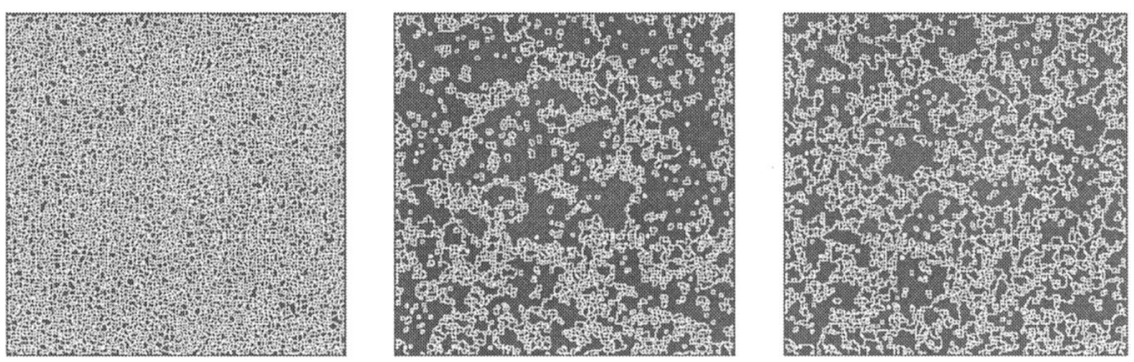

Fig. 13. Uniform noise: (a) $\mathrm{SNR}=12.2 \mathrm{db}\left\{\sigma^{2}=6\right\}$, (b) $\mathrm{SNR}=10.0 \mathrm{db}\left\{\sigma^{2}=10\right\}$, (c) $\mathrm{SNR}=8.2 \mathrm{db}\left\{\sigma^{2}=15\right\}$, (d) $\mathrm{SNR}=6.9 \mathrm{db}$ $\left\{\sigma^{2}=20\right\}$. First column: squared gradient image. Second column: oversegmented image. Third column: optimum level by applying algorithm (DC). Fourth column: optimum level by applying algorithm (MCPs).

segments to a contour representation based on Catmull-Rom splines [8] which enables a further flexible manual adjustment in those regions where the border of the object is not respected (Fig. 18 - bottom right view). In this approach parts of the object, which are very smooth, are characterized by only a few points, while jagged edges can be approximated at any accuracy by addition or dragging points to the edges with immediate update of the spline-contour. A contour represented by 


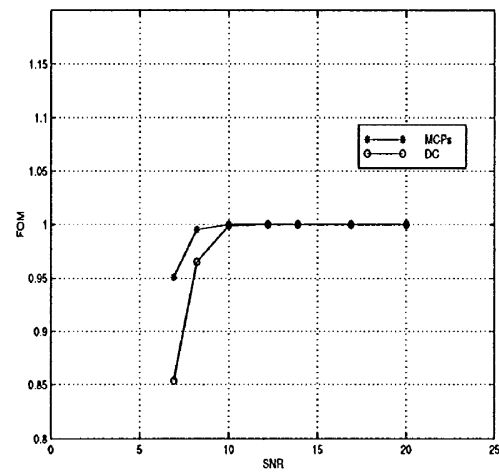

(a)

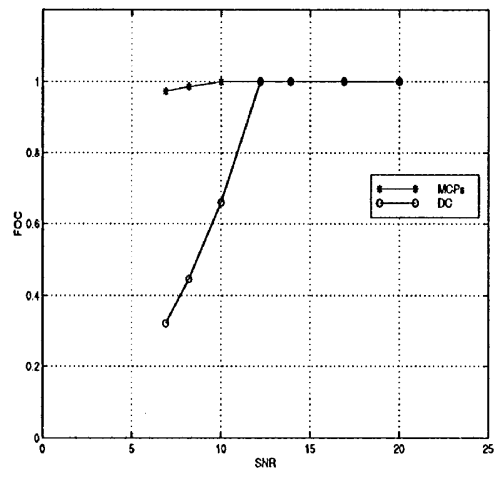

(b)

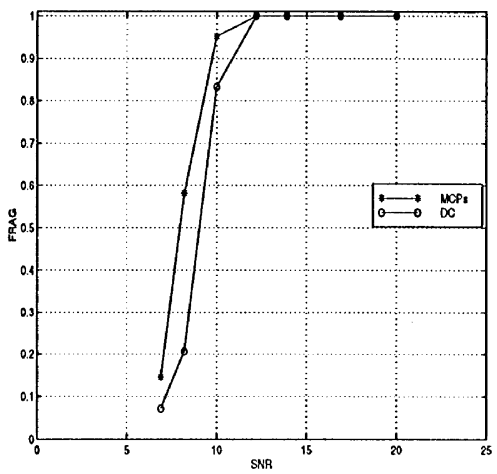

(c)

Fig. 14. (a) FOM, (b) FOC, (c) FRAG (Gaussian noise).

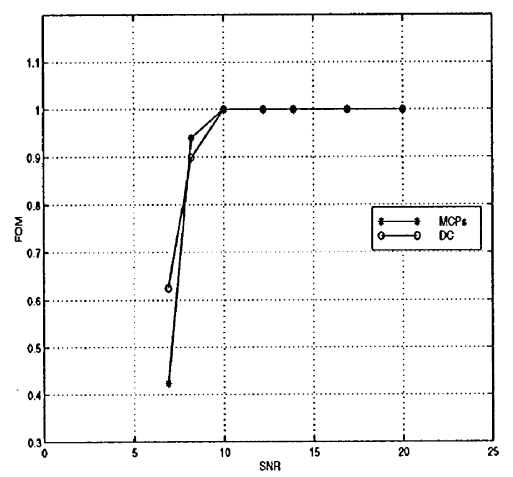

(a)

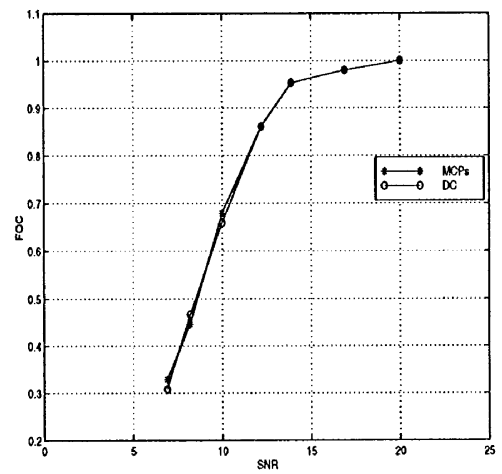

(b)

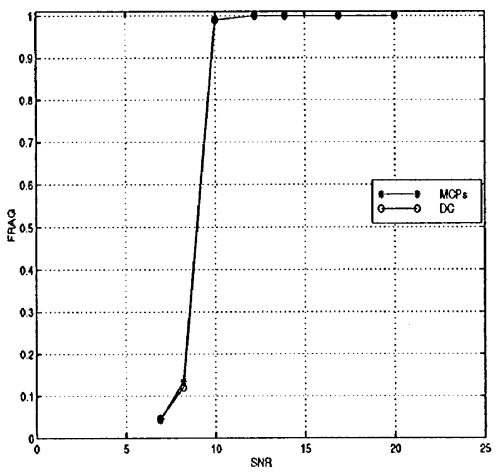

(c)

Fig. 15. (a) FOM, (b) FOC, (c) FRAG (Uniform noise).

Catmull-Rom splines, which belong to the family of cardinal splines, passes through all points marked by the user, which is not the case for representations based on B-splines or Bezier curves where a distinction is made between the points the curve is passing through and the control knots, which allow direct control of the slopes of the curve. Definition of these control knots under the constraint that the spline should follow the trace of a visible edge makes manual delineation less efficient.

During the editing of the contour, the consistency of the object in 3D can be checked by looking at the cross-section of the contour in the other orthogonal planes (axial, coronal or sagittal). Once the user is completely satisfied, the contour is added to the stack of contours of the object and converted to a $3 \mathrm{D}$ voxel representation of the object, which is scanned in real-time in order to retrieve and show the updated outlines of the object in the other orthogonal planes (Fig. 18).

The whole concept has been integrated into a 3D modality editor software, aimed at the creation of reference sets of anatomical objects (i.e. digital atlases) and morphometrical studies [6]. The method is currently applied to brain MR images in order to measure the cerebellar volume of children. Regarding the speed of the whole procedure, we report that a complete stack of hierarchical levels for one slice can be obtained after an average processing time of $20 \mathrm{~s}$. This duration is dependent on the initial 


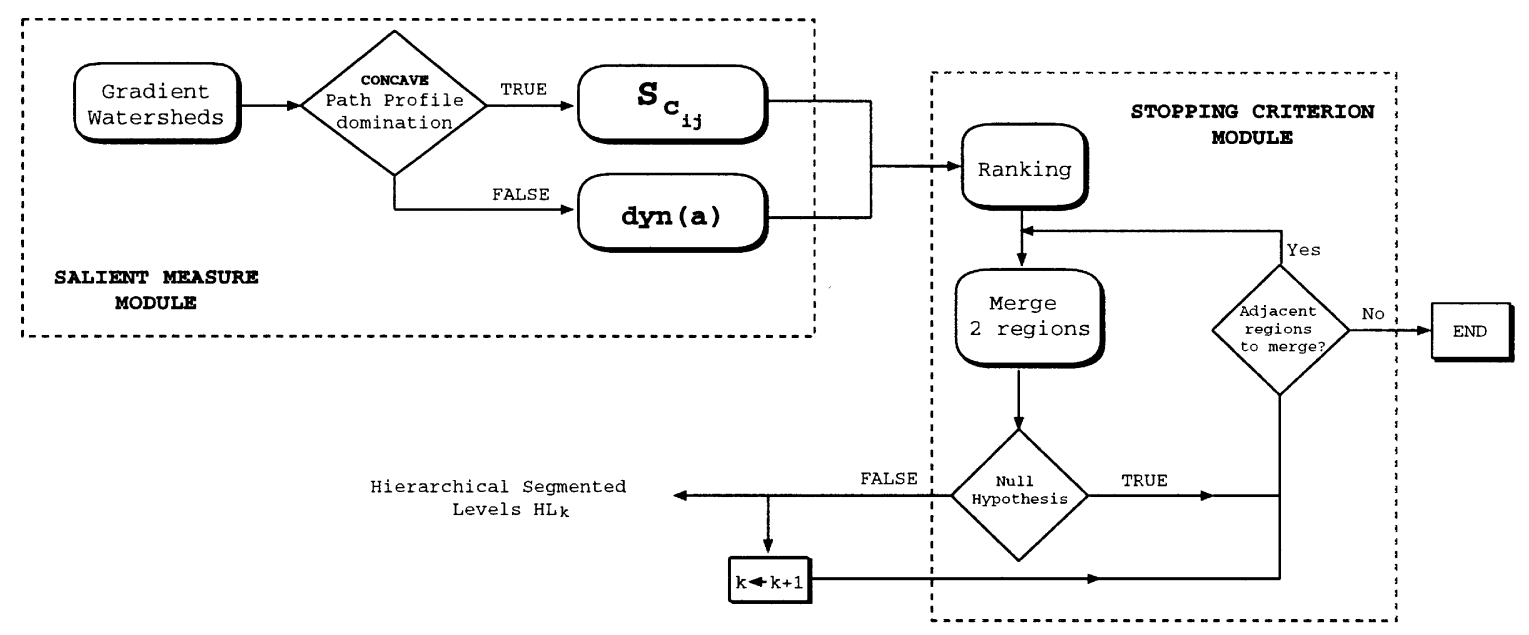

Fig. 16. The proposed hierarchical segmentation scheme.

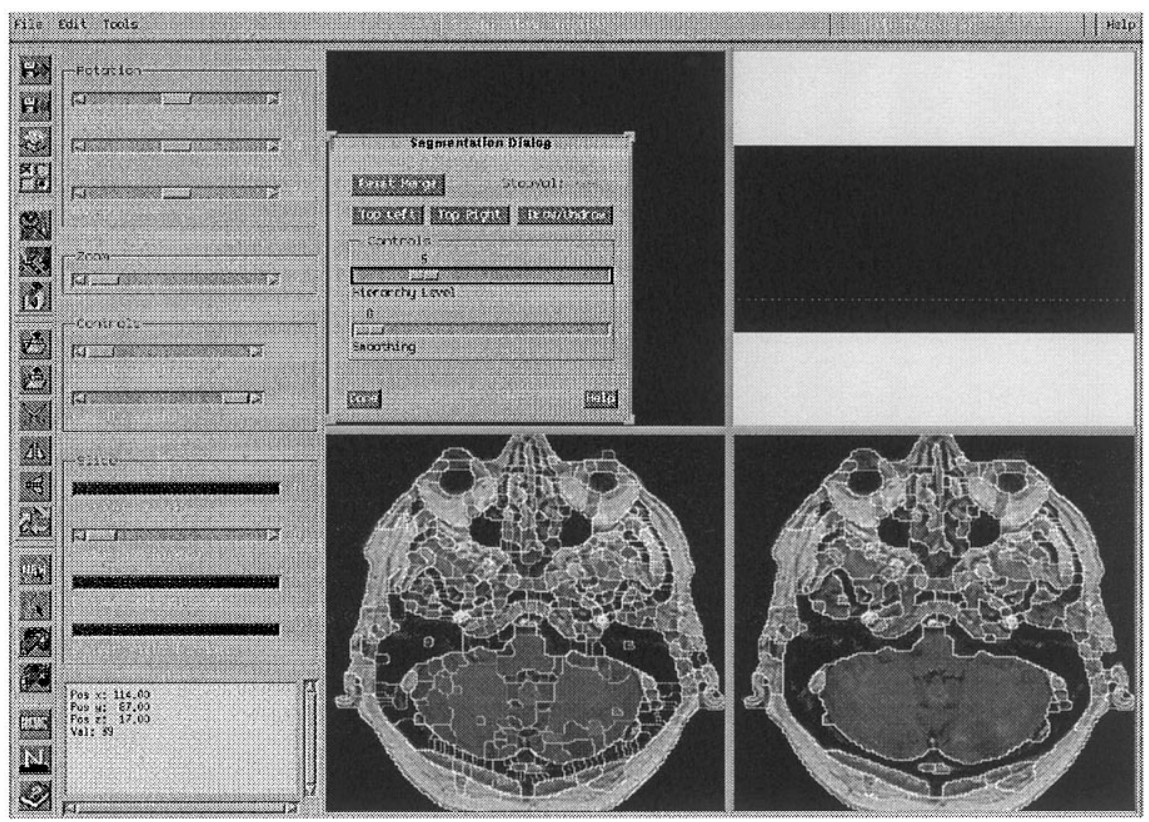

Fig. 17. Browsing among hierarchical levels.

number of regions, as they are obtained by the gradient watersheds. Eventually, this computation time has not to be considered as a temporal load for each slice since for every slice next to the first the computation may turn to a background process.
This actually compensates the time spent for any manual correction. The accuracy of our method was demonstrated in the volume measurement of the cerebellum. We compared the volume obtained from a series of manually delineated contours and 


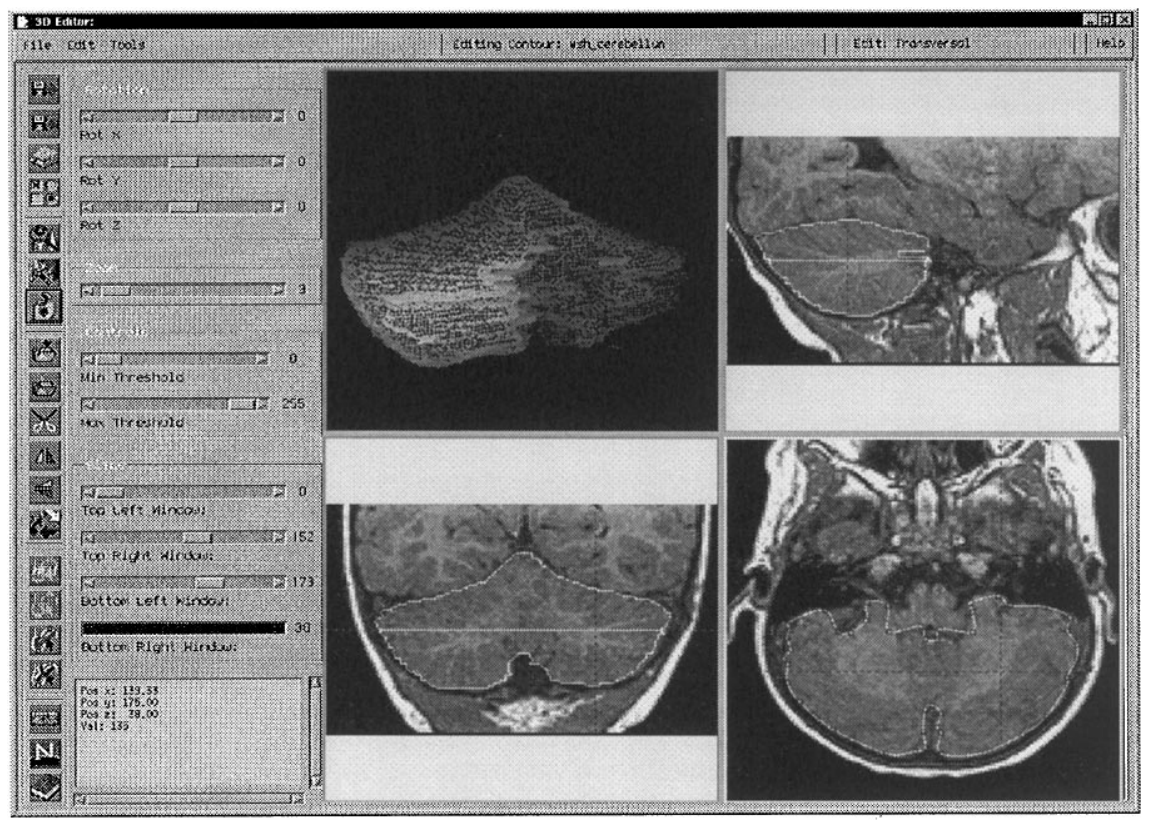

Fig. 18. Top left view: wiremesh of the $3 \mathrm{D}$ delineated contour set. Other views: simultaneous $3 \mathrm{D}$ orthogonal viewing for interactive correction.

the one obtained by our method for the same set of images. The pure manual method identified 121996 voxels (resolution $0.966 \mathrm{~mm}^{3}$ ) which resulted in a volume of $109.97 \mathrm{~cm}^{3}$. Our method identified 119935 voxels which corresponds to $108.112 \mathrm{~cm}^{3}$.

\section{Conclusion}

In this work, we create a hierarchy among the gradient watersheds meeting the requirements (i) of preserving the topology of the initial watershed lines and (ii) of extracting homogeneous objects at a larger scale. We study the construction of hierarchies for gradient watersheds retrieving information from the superficial image structure. Basically, the underlying analysis is based on the fact that the gradient image provides information with respect to local discontinuity and similarity. Two algorithms which utilize this type of information are analyzed. The first one is the dynamics of contours [16] and the second is the algorithm which considers the minimum cost path saliency [21]. For both algorithms, a stopping criterion which defines and extracts automatically each hierarchical level is proposed. An important hint for the superior behavior of one of the two algorithms is the domination of the profile type of the surface formed by the gradient image. Experiments indicate that when convex path profiles dominate, the Constrained $d y$ namics of contours demonstrates the best behavior. On the other hand, if concave path profiles dominate then the Minimum cost path saliency performs at best. Therefore, both algorithms need to be available, and be chosen according to the path profile domination as discussed. The experimental results which are discussed correspond to both artificial and real images. Our bottom-up procedure provides an essential aid to the precise delineation and classification of anatomical objects when used together with a user-interface environment. The user task is made easier by the heavy reduction of the number of regions obtained after the application of the hierarchical scheme. In short, the reported study has contributed to the following:

1. It provides an optimum solution for the construction of a hierarchy for the gradient watershed adjacent groups. 
2. It improves the concept of dynamics of contours due to the provided ability to retrieve automatically different hierarchical levels.

3. It facilitates interactivity for the delineation and labeling of anatomical structures.

\section{Appendix A. A tensor for the Chamfer metric (a-b) description}

Definition A.1. Prime vectors are the elements of a set of vectors $V=\left(u_{1}, u_{2}, \ldots, u_{k}\right)$ in $Z^{2}$, such that if $u \in V$ then it is implied that $-u \in V$.

Definition A.2. If $x$ and $y$ are two points in $Z$, a path from $x$ to $y$ is defined as a sequence $p_{0}, p_{1}, \ldots, p_{k}$ of points such that $p_{0}=x$ and $p_{k}=y$ and such that $p_{i}-p_{i-1} \in V$ for $i=1, \ldots, k$.

Definition A.3. The length of this path is defined as: $L\left(p_{0}, p_{1}, \ldots, p_{k}\right)=\sum_{i=1}^{k} l\left(p_{i}-p_{i-1}\right)$. If $x=y$, we consider $p_{0}=x=y$ as a path from $x$ to $y$. The length of this path is 0 .

Definition A.4. Let $v$ and $w$ be two independent vectors in $R^{2}$. The wedge between these two vectors is the set

$$
W_{v, w}=a_{1} v+a_{2} w \mid a_{1}, a_{2} \in R^{+} .
$$

Definition A.5. Let $V=\left(u_{1}, u_{2}, \ldots, u_{k}\right)$ be a set of prime vectors, two independent vectors $v, w \in V$ are adjacent if $W_{v, w}$ contains no other prime vectors than $v$ and $w$.

Lemma A.1. Any Chamfer metric $a-b(a \leqslant b)$ can be described by the tensor

$g_{i j}^{\prime}(\boldsymbol{x})=\left[\begin{array}{cc}2 / b^{2} & 1 / a b \\ 1 / a b & 1 / a^{2}\end{array}\right]$.

Proof. Suppose that $\vec{u}_{1}, \vec{u}_{2}$ are adjacent prime vectors. Lemma 3.7 of [14] states that each grid point in a wedge can be written as an integer combination of the two prime vectors generating this wedge. This also immediately produces a path from the origin to each point in which only two prime vec- tors occur as steps. Because of invariance with respect to translation of the origin to one of the points $x, y \in Z^{2}$, it follows that for each $x, y$ there is a path from $x$ to $y$ that contains at most two different prime vectors as steps. In [14] has been proved that this is a shortest path. The above conclusion makes it adequate to get an expression for the tensor $g_{i j}^{\prime}$ by computing it at any octant (wedge), created by the adjacent prime vectors $\vec{u}_{i}$. Let $\vec{e}_{1}, \vec{e}_{2}$ denote two orthonormal vectors which assign the orthonormal coordinate system, see Fig. 19.

Combination with adjacent prime vectors yields

$a \vec{u}_{2}=\vec{e}_{2}$

$b \vec{u}_{1}=\vec{e}_{1}+\vec{e}_{2}$

A point $P$ at the second octant (see Fig. 19), can be expressed by a vector $\vec{V}$, as follows:

$$
\begin{aligned}
\vec{V}=x \vec{e}_{2}+y \vec{e}_{1} & =x a \vec{u}_{2}+y b \vec{u}_{1}-y a \vec{u}_{2} \\
& =y b \vec{u}_{1}+x a \vec{u}_{2}-y a \vec{u}_{2} \\
& =m_{1} \vec{u}_{1}+m_{2} \vec{u}_{2},
\end{aligned}
$$

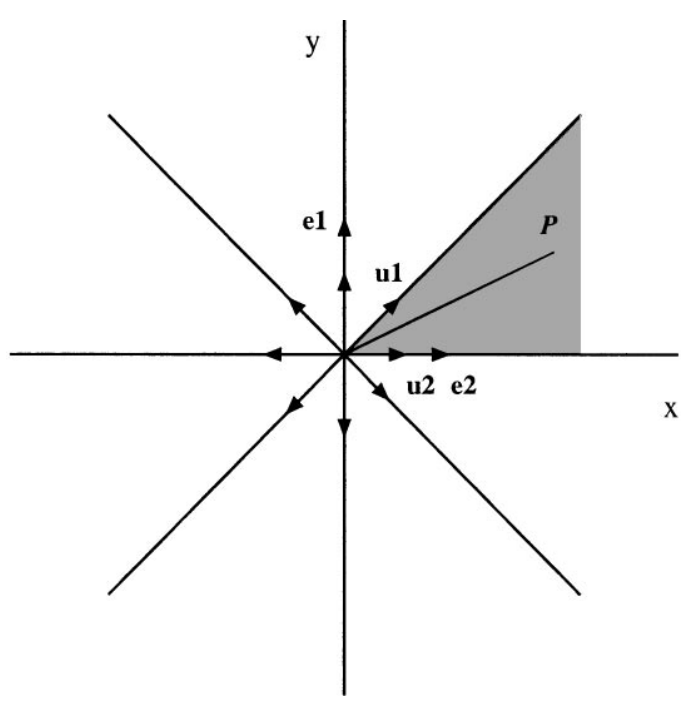

Fig. 19. A point $P$ inside the wedge $W_{u_{1} u_{2}}$ with adjacent prime vectors $\vec{u}_{1}, \vec{u}_{2}$. 
$m_{1}=y b \Rightarrow y=\frac{m_{1}}{b}$,

$m_{2}=x a-y a \Rightarrow x=\frac{m_{2}}{a}+\frac{m_{1}}{b}$.

Considering that $\delta_{i j}=g_{i j}=\left[\begin{array}{ll}1 & 0 \\ 0 & 1\end{array}\right]$, we get

$g_{11}^{\prime}=\frac{\partial x}{\partial m_{1}} \frac{\partial x}{\partial m_{1}} g_{11}+\frac{\partial y}{\partial m_{1}} \frac{\partial y}{\partial m_{1}} g_{22}=\frac{1}{b^{2}}+\frac{1}{b^{2}}=\frac{2}{b^{2}}$

$g_{12}^{\prime}=\frac{\partial x}{\partial m_{1}} \frac{\partial x}{\partial m_{2}} g_{11}+\frac{\partial y}{\partial m_{1}} \frac{\partial y}{\partial m_{2}} g_{22}=\frac{11}{b a}+0=\frac{1}{a b}$,

$g_{21}^{\prime}=\frac{\partial x}{\partial m_{2}} \frac{\partial x}{\partial m_{1}} g_{11}+\frac{\partial y}{\partial m_{2}} \frac{\partial y}{\partial m_{1}} g_{22}=\frac{11}{a b}+0=\frac{1}{a b}$,

$g_{22}^{\prime}=\frac{\partial x}{\partial m_{2}} \frac{\partial x}{\partial m_{2}} g_{11}+\frac{\partial y}{\partial m_{2}} \frac{\partial y}{\partial m_{2}} g_{22}=\frac{11}{a a}+0=\frac{1}{a^{2}}$

$g_{i j}^{\prime}=\left[\begin{array}{cc}\frac{2}{b^{2}} & \frac{1}{a b} \\ \frac{1}{a b} & \frac{1}{a^{2}}\end{array}\right]$,

\section{References}

[1] J.-M. Beaulieu, M. Goldberg, Hierarchy in picture segmentation: A stepwise optimization approach, IEEE Trans. Pattern Anal. Machine Intell. 11 (2) (1989) 150-163.

[2] S. Beucher, Segmentation d'mages et morphologie mathématique, $\mathrm{PhD}$ thesis, Ecole Nationale Supérieure des Mines de Paris, Fontainebleau, 1990.

[3] S. Beucher, Watershed, hierarchical segmentation and waterfall algorithm, in: J. Serra, P. Soille (Eds.), Mathematical Morphology and its Applications on Image and Signal Processing, Kluwer Academic Publishers, Dordrecht, 1994, pp. 69-76.

[4] S. Beucher, F. Meyer, The morphological approach to segmentation: The watershed transformation, in: E.R. Dougherty (Ed.), Mathematical Morphology in Image Processing, Marcel Dekker, New York, 1993, Chapter 12, pp. 433-481.

[5] G. Borgefors, Distance transformations in digital images, Comput. Vision Graph. Image Process. 34 (1986) 344-371.

[6] R. Deklerck, A. Salomie, SAMMIE HC 1044 (HC) - Deliverable (05) D3.1 - BrainWorks I, Part III: The 3D
Modality Editor, August 1996. Available at http://etro. vub.ac.be/projects/hc_sammie/3d_editor/EdHlp.html.

[7] M. Grimaud, A new measure of contrast: The dynamics, in: Image Algebra and Morphological Image Processing III, SPIE, Vol. 1769, San Diego, CA, 1992, pp. 292-305.

[8] D. Hearn, M.P. Baker, Computer Graphics, Prentice-Hall, Englewood Cliffs, NJ, 1994.

[9] J.J. Koenderink, The structure of images, Biol. Cybernet. 50 (1984) 363-370.

[10] L.M. Lifshitz, S.M. Pizer, A multiresolution hierarchical approach to image segmentation based on intensity extrema, IEEE Trans. Pattern Anal. Machine Intell. 12 (6) (1990) 529-541.

[11] T. Lindeberg, Scale Space Theory in Computer Vision, Kluwer Academic Publishers, Dordrecht, The Netherlands, 1994.

[12] F. Maes, D. Vandermeulen, P. Suetens, G. Marchal, Automatic image partitioning for generic object segmentation in medical images, in: Y. Bizais et al. (Eds.), Information Processing in Medical Imaging, Kluwer Academic Publishers, Dordrecht, The Netherlands, 1995, pp. $215-226$.

[13] F. Meyer, Topographic distance and watershed lines, Signal Processing 38 (1994) 113-125.

[14] P.F.M. Nacken, Chamfer metrics in mathematical morphology, J. Math. Imaging Vision 4 (1994) 233-253.

[15] L. Najman, M. Schmitt, Watershed of a continuous function, Signal Processing 38 (1994) 99-112.

[16] L. Najman, M. Schmitt, Geodesic saliency of watershed contours and hierarchical segmentation, IEEE Trans. Pattern Anal. Machine Intell. 18 (12) (1996) 1163-1173.

[17] A. Papoulis, Probability, Random Variables and Stochastic Processes, McGraw-Hill, Singapore, 1991.

[18] S.M. Pizer, T.J. Cullip, R.E. Fredericksen, Toward interactive object definition in 3D scalar images, in: K.H. Hohne et al. (Ed.), 3D Imaging in Medicine, NATO ASI Series, Springer, Berlin, 1990, Vol. F60, pp. 83-105.

[19] I.E. Pratikakis, Hierarchy determination of the gradient watershed adjacent groups - the superficial structure, Technical Report 44, Vrije Universiteit Brussel - ETRO/IRIS, November 1997.

[20] I.E. Pratikakis, R. Deklerck, A. Salomie, J. Cornelis, Improving precise interactive delineation of $3 \mathrm{D}$ structures in medical images, in: H.U. Lemke (Ed.), Computer Assisted Radiology, Berlin, 1997, pp. 215-220.

[21] I.E. Pratikakis, H. Sahli, J. Cornelis, Hierarchy determination of the gradient watershed adjacent groups, in: 10th Scandinavian Conf. on Image Analysis, Lappeenranta, Finland, 1997, pp. 685-692.

[22] W.K. Pratt, Digital Image Processing, Wiley, New York, 1978.

[23] D. Rutovitz, Datastructures for operations on digital images, in: D.K. Pollock, G.C. Cheng, R.S. Ledley, A. Rosenfeld (Eds.), Pictorial Pattern Recognition, Thomson, Washington, DC, 1968, pp. 105-133. 
[24] P. Soille, Generalized geodesic distances applied to interpolation and shape description, in: J. Serra, P. Soille (Eds.), Mathematical Morphology and its Applications on Image and Signal processing, Kluwer Academic Publishers, Dordrecht, 1994, pp. 193-200.

[25] M. Spivak, A Comprehensive Introduction to Differential Geometry, Perish, Boston, 1979.

[26] K.C. Strasters, Quantitative analysis in confocal image cytometry, PhD Thesis, Technical University of Delft, 1994.
[27] P.W. Verbeek, B.J.H. Verwer, Shading from shape, the eikonal equation solved by grey-weighted distance transform, Pattern Recognition Lett. 11 (1990) 681-690.

[28] B.J.H. Verwer, P.W. Verbeek, S.T. Dekker, An efficient uniform cost algorithm applied to distance transforms, IEEE Trans. Pattern Anal. Machine Intell. 11 (4) (1989) 425-429. 